ALEA, Lat. Am. J. Probab. Math. Stat. 18, 1773-1799 (2021)

DOI: $10.30757 /$ ALEA.v18-66

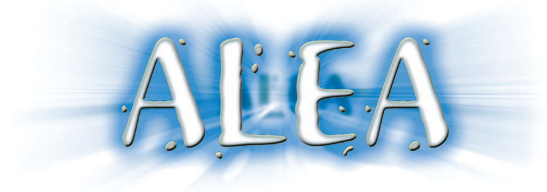

\title{
A combinatorial representation for the invariant measure of diffusion processes on metric graphs
}

\author{
Michele Aleandri, Matteo Colangeli and Davide Gabrielli \\ Università LUISS Guido Carli, Viale Romania, 32, 00197 Roma, Italia. \\ E-mail address: maleandri@luiss.it \\ Università dell'Aquila, Via Vetoio, Loc. Coppito, 67010 L'Aquila, Italia. \\ E-mail address: matteo.colangeli1@univaq.it \\ Università dell'Aquila, Via Vetoio, Loc. Coppito, 67010 L'Aquila, Italia. \\ E-mail address: davide.gabrielli@univaq.it
}

\begin{abstract}
We study a generalization to a continuous setting of the classical Markov chain tree theorem. In particular, we consider an irreducible diffusion process on a metric graph. The unique invariant measure has an atomic component on the vertices and an absolutely continuous part on the edges. We show that the corresponding density at $x$ can be represented by a normalized superposition of the weights associated to metric arborescences oriented toward the point $x$. A metric arborescence is a metric tree oriented towards its root. The weight of each oriented metric arborescence is obtained by the product of the exponential of integrals of the form $\int \frac{b}{\sigma^{2}}$, where $b$ is the drift and $\sigma^{2}$ is the diffusion coefficient, along the oriented edges, for a weight for each node determined by the local orientation of the arborescence around the node and for the inverse of the diffusion coefficient at $x$. The metric arborescences are obtained by cutting the original metric graph along some edges.
\end{abstract}

\section{Introduction}

A powerful construction for finite state Markov chains is the so-called Markov Chain Matrix Tree Theorem (Anantharam and Tsoucas, 1989; Freidlin and Wentzell, 2012; Pitman and Tang, 2018). In the case of an irreducible continuous time finite state Markov chain the unique invariant measure is obtained as a normalized superposition of weights associated to some combinatorial structures. The combinatorial structures considered are the rooted arborescences of the transition graph. A rooted arborescence of a directed graph is a directed spanning tree-like subgraph oriented towards a single vertex $x$, called the root (see the precise definition 2.1). The transition graph of the chain is a directed graph with vertices corresponding to the states and directed edges corresponding to the possible transitions. For an irreducible chain the graph is strongly connected. The weight of each

Received by the editors June 8th, 2020; accepted August 11th, 2021.

2010 Mathematics Subject Classification. 60G10, 60J60, 60C05.

Key words and phrases. Diffusion processes, metric graphs, stationarity. 
arboresence is the product of the rates of all the edges that it contains. The invariant measure at $x$ coincides with the normalized sum of the weights of all arborescences rooted at $x$.

A classical result in probability theory is the diffusive rescaling of a class of random walks with convergence to a diffusion process. The prototype of this class of results is the celebrated Donsker's Theorem. A diffusive random walk is obtained, generically, by weakly perturbing a reversible random walk. The most general reversible random walk on a graph is determined by some positive weights associated to vertices and symmetric positive weights associated to the edges. In the scaling limit, we consider a grid of mesh (i.e. the distance between nearest neighbors vertices) $1 / N$ embedded into $\mathbb{R}^{d}$ and consider the weights on the vertices and edges as discretized versions of smooth positive functions that we call $\alpha$ and $Q$ respectively. Likewise, the weak perturbation is obtained by the discretization of a smooth vector field $F$. The family of diffusive walks is therefore parameterized by the triple $(\alpha, Q, F)$. Correspondingly, the family of limiting processes, which are the diffusion processes, are parameterized by the pair $(b, \sigma)$ (see equation (3.1)) where the vector field $b$ is called the drift and the matrix $\sigma$ is called the diffusion coefficient. The correspondence between $(\alpha, Q, F)$ and $(b, \sigma)$ is not one-to-one and a whole class of microscopic models converge to the same diffusion process.

A very natural question is whether the combinatorial representation of the invariant measure for the random walks has a corresponding continuous version for the diffusion processes. We answer this question positively in the case of diffusions on metric graphs. A metric graph is a metric space that is obtained by gluing the extrema of a finite number of bounded segments to some vertices. A diffusion process on a metric graph is a process that evolves like a diffusion along each edge and then, when reaching a vertex, it evolves by possibly spending some random time therein and by then picking up at random a new edge upon which the evolution continues.

We prove in this paper that the invariant measure of a diffusion process on a metric graph has a representation that is the continuous counterpart of the combinatorial representation of the matrix tree theorem. More precisely we have that the density of the invariant measure at a point $x$ belonging to an edge is obtained as follows. Given the metric graph we can obtain a metric tree by cutting some edges. Each edge can be cut in different ways at a point parameterized by a real parameter. From the metric tree we obtain a metric arborescence rooted at $x$ by simply orienting all the edges towards $x$. To each metric arborescence we associate a weight. The weight is obtained as the product of several terms, one for each edge and one for each vertex. The weight on an edge is given by the exponential of $\int s_{\mathbf{e}}$ where the integral is an integral along the edge $\mathbf{e}$ according to its orientation on the arborescence and $s_{\mathbf{e}}:=\frac{b_{\mathbf{e}}}{\sigma_{\mathbf{e}}^{2}}$, where $b_{\mathbf{e}}$ and $\sigma_{\mathbf{e}}^{2}$ are the drift and the diffusion coefficients on the metric edge e. The weight associated to each node depends on the local orientation of the arborescence around the vertex (all apart one edge are oriented entering into the vertex) and the parameters describing the stochastic evolution at the node. In the continuous setting there is an extra factor, associated with the root $x$, that appears in the weight of each arborescence and is given by $\sigma_{\mathbf{e}}^{-2}(x)$. The value of the invariant measure at each node is then adjusted depending again on the behaviour of the model on the vertices.

The strategy of the proof is the following. Before we compute the scaling limit of the combinatorial construction of the matrix tree theorem for one dimensional diffusive random walks on a ring. We obtain that, for any triple $(\alpha, Q, F)$ that correspond to the same $(b, \sigma)$, the limit is the same and coincides with the continuous construction above described. In this case a metric arborescence is obtained from the ring by just one single cut. The proof of this universal scaling limit is short and informal. For a general metric graph we obtain a generalization of this formula involving integrations over a finite number of cut points. The number of cuts depends on the geometric structure of the graph. The proof in the general case is instead obtained extending in a natural way the formula for the ring to a general metric graph and then using the characterization of the invariant measure in terms of the generator of the process. 
There is a large literature concerning stochastic processes on metric graphs, see for example Freidlin and Sheu (2000); Freidlin and Wentzell (1993); Hajri and Raimond (2014); Kostrykin et al. (2012). Models with equations and processes defined on metric graphs are used in several different applicative frameworks, like quantum mechanics (Berkolaiko et al., 2006), traffic flow (Garavello and Piccoli, 2006) and many others (Berkolaiko et al., 2006). Our representation could be very useful in studying weak noise asymptotics (Freidlin and Wentzell, 2012) of diffusions on metric graphs and in this limit it is very strongly connected with Hamilton Jacobi equations on metric graphs (Camilli and Marchi, 2013).

We discuss the problem in a geometric framework that consists of several one dimensional spaces non-trivially glued. We believe that a continuous version of the combinatorial construction exists in much more general frameworks, as for example for domains in $\mathbb{R}^{d}$ with $d \geq 2$. In this sense this paper is a first step towards a proof of an equation of the form

$$
\mu(x)=\frac{1}{\sigma^{2}(x) Z} \int_{\Omega} \mathcal{D}\left(\tau_{x}\right) e^{\int_{\Omega}\left(b(y), \sigma^{-2}(y) v(y)\right)} .
$$

In the above equation $\Omega$ is a domain of $\mathbb{R}^{d}$ and $x \in \Omega ; \int \mathcal{D}\left(\tau_{x}\right)$ is an integration over arborescenses rooted at $x$, the vector $v(y)$ denotes the direction at $y$ of the arborescence $\tau_{x}$ and $(\cdot, \cdot)$ denotes the Euclidean scalar product. A challenge here is to give a meaning to all these objects. The generalization to different state spaces and different Markov processes is also interesting and even more challenging.

There are also other natural and interesting issues to be discussed in the framework of metric graphs. These are for example: a determinantal representation and interpretation of the formulas, like in the discrete case; the relationship with the discrete time Markov chain obtained observing just the sequence of vertices visited, see e.g. Fitzsimmons and Kuter (2015), Jehring (2009); the connection with the theory of electrical networks and harmonic functions on metric graphs, see respectively Berkolaiko et al. (2006), Fitzsimmons and Kuter (2015). We are not going to discuss here these issues.

The paper is organized as follows.

In Section 2 we shortly recall the classic Markov chain matrix tree theorem for finite state irreducible continuous time Markov chains.

In Section 3 we discuss briefly one dimensional diffusion processes. Then we recall the scaling limit of diffusive random walks giving the relation between the microscopic triple $(\alpha, Q, F)$ and the macroscopic pair $(b, \sigma)$. We discuss the scaling limit of the discrete arborescences and the corresponding weights obtaining formulas written in terms of continuous metric arborescences. Finally, we show by a direct computation that the representations obtained in the case of the circle and the interval give the correct result.

In Section 4 we describe shortly the metric graphs.

In Section 5 we discuss the definition of a diffusion process on a metric graph. This is done by exhibiting the form of the generator that depends on a collection of parameters related to the behaviour of the process in correspondence of the vertices.

In Section 6 we prove the validity of the representation formula for the invariant measure of a diffusion on a metric graph in terms of a normalized sum of weights associated with continuous metric arborescences obtained by cutting the original metric graph at a finite number of points. We show moreover that the reversibility condition corresponds to the reversibility of a finite state effective Markov chain evolving on the vertices of the graph.

\section{Markov chain tree Theorem}

Here we briefly recall a classic representation of the invariant measure of a finite irreducible Markov chain (see for example Freidlin and Wentzell, 2012 or Pitman and Tang, 2018 for a recent 
general overview or Anantharam and Tsoucas, 1989 for a simple proof). The general framework is the following. We consider a Markov chain having transition graph $(V, E)$. The finite set $V$ is the state space while the set of directed edges $E$ represents the collection of possible transitions of the chain. If $(x, y) \in E$ we have that the rate $r(x, y)$ of jump from $x$ to $y$ is strictly positive. We say that the directed edge $(x, y)$ exits from $x$ and enters into $y$.

The stationarity condition that the invariant measure $\pi$ has to satisfy is given by

$$
\pi(x) \sum_{y:(x, y) \in E} r(x, y)=\sum_{y:(y, x) \in E} \pi(y) r(y, x), \quad \forall x \in V .
$$

If the transition graph $(V, E)$ is strongly connected (any two points can be connected by directed paths) then the chain is irreducible and the invariant measure is unique and strictly positive Norris (1998). We restrict to this case.

Definition 2.1. Let $(V, E)$ be a directed graph. An arborescence $\tau$ directed toward $x \in V$ is a spanning subgraph of $(V, E)$ such that:

1) For each vertex $y \neq x$ there is exactly one directed edge exiting from $y$ and belonging to $\tau$;

2) For any $y \in V$ there exists one directed path from $y$ to $x$ in $\tau$;

3) There are no edges exiting from $x$.

Let $\mathcal{T}_{x}$ the set of arborescences of $(V, E)$ directed toward $x \in V$.

If the chain is irreducible, then $\mathcal{T}_{x}$ is not empty for any $x$.

Equivalently the arborescences in $\mathcal{T}_{x}$ can be characterized as follows. Take the transition graph $(V, E)$ and construct the corresponding undirected graph $(V, \mathcal{E})$ obtained simply transforming each directed edge into an undirected one and removing all the multiple undirected edges obtained. Indeed we can have multiple undirected edges since more than one single directed edge may have the same extremal vertices. We allow no more than one single undirected edge connecting two vertices. An element $\tau \in \mathcal{T}_{x}$ is characterized by the fact that if we ignore orientation of the edges of $\tau$ we obtain a spanning tree of $(V, \mathcal{E})$. Moreover, any directed edge in $\tau$ exiting from $y \neq x$ is directed according to the orientation obtained going along the unique unoriented path in the tree going from $y$ to $x$.

To any arborescence $\tau$ we associate a weight given by

$$
R(\tau):=\prod_{e \in \tau} r(e)
$$

where $r: E \rightarrow(0, \infty)$ are the transition rates. In the above formula the product is over all the directed edges $e$ that are edges of the arborescence $\tau$.

The Markov chain matrix tree Theorem claims that the invariant measure of the chain is given by

$$
\mu(x)=\frac{\sum_{\tau \in \mathcal{T}_{x}} R(\tau)}{\sum_{z \in V} \sum_{\tau \in \mathcal{T}_{z}} R(\tau)} .
$$

See Pitman and Tang (2018) for a interpretation in terms of determinants of (2.3).

\section{One dimensional diffusions}

Let $I$ be a finite interval in $\mathbb{R}$ and let us consider a one dimensional diffusion process determined by a stochastic differential equation

$$
d X(t)=b(X(t)) d t+\sigma(X(t)) d W(t)
$$

where $b$ is a $C^{1}$-Lipschitz function, called the drift, and $\sigma>0$ is a strictly positive $C^{2}$-Lipschitz function, called the diffusion coefficient. Under these assumptions we have that the invariant measure $\mu=\mu(x) d x$ has a $C^{2}$-density (this is a consequence of the following explicit formulas but see 
also Sections 4.1, 4.2 and 4.3 of Pavliotis, 2014) that is a strong solution to

$$
\frac{1}{2} \partial_{x}^{2}\left(\sigma^{2}(x) \mu(x)\right)-\partial_{x}(b(x) \mu(x))=0,
$$

where $x$ belongs to the interior of $I$. We assume strong regularity of the coefficients since we concentrate on the geometric construction of the invariant measures and try to minimize the unrelated technical details. Equation (3.2) can be naturally written as follows. Consider a measure with $C^{2}$-density $\nu=\nu(x) d x$ and define the corresponding probability current as

$$
J(\nu):=-\frac{1}{2} \partial_{x}\left(\sigma^{2}(x) \nu(x)\right)+b(x) \nu(x) .
$$

The stationarity condition for the invariant measure $\mu$ can be written in terms of the current (3.3) in one of the two equivalent conditions:

Let us write

$$
\begin{array}{cc}
\text { i) } & \partial_{x} J(\mu)=0 \\
\text { ii) } & J(\mu)=\text { constant . }
\end{array}
$$

$$
s(x):=\frac{b(x)}{\sigma^{2}(x)} ; \quad S(x):=2 \int_{x^{*}}^{x} s(y) d y,
$$

where $x^{*}$ is an arbitrary point. The general solution to (3.2) on $\mathbb{R}$ is

$$
\mu(x)=\frac{1}{\sigma^{2}(x)}\left[k_{1}+k_{2} \int_{x^{*}}^{x} e^{-S(y)} d y\right] e^{S(x)}
$$

where $k_{i}$ are constants that have to be determined in order to obtain the invariant measure of (3.1). This procedure depends on the particular geometric framework and boundary conditions that we consider. Within the class of stationary measures which satisfy Eq. (3.2), a special mention goes to the so-called equilbrium measures which characterize reversible processes, and for which $J(\mu)=0$ holds. This case is tackled in the next example and, more extensively, also in Theorem 6.3 below.

Example 1: Let us first consider the special case when the process (3.1) is defined on an interval $[a, b]$ with reflecting boundary conditions (see forthcoming Section 5 for a detailed discussion of boundary conditions). Since there is no flow across the boundaries, the stationarity condition (3.4) coincides with $J(\mu)=0$, the process is always reversible and we easily get

$$
\mu(x)=\frac{e^{S(x)}}{Z \sigma^{2}(x)},
$$

where $Z$ is a normalization factor. This means that we have to fix in (3.6) $k_{2}=0$ and the value of $k_{1}$ is then fixed by the normalization condition.

Example 2: Consider now the process (3.1) on a ring of length one $\mathcal{S}^{1}:=\mathbb{R} / \mathbb{Z}$. This is equivalent to fixing the coefficients in (3.1) to be periodic with period one. Given $z \in \mathbb{R}$ we denote by $\pi(z) \in \mathcal{S}^{1}$ its projection. We draw $\mathcal{S}^{1}$ as a ring on which the anticlockwise direction corresponds to the direction of the motion of $\pi(z+t)$ for increasing $t$.

Given $x \neq y \in \mathcal{S}^{1}$ we call $I^{ \pm}[x, y]$ the closed intervals containing the points of $\mathcal{S}^{1}$ encountered moving on the ring from $x$ to $y$ respectively anticlockwise for the + sign and clockwise for the sign.

We will use integrals over oriented intervals of $\mathcal{S}^{1}$ so that our intervals will be always oriented. In particular the intervals $I^{ \pm}[x, y]$ have always the orientations from $x$ to $y$. We have therefore that $I^{+}[x, y]$ and $I^{-}[y, x]$ have the same support but opposite orientations since $I^{+}[x, y]$ is anticlockwise oriented while $I^{-}[y, x]$ is clockwise oriented. When we write $\mathcal{S}^{1}$ we mean always the ring anticlockwise oriented.

Consider $I$ an oriented interval of $\mathcal{S}^{1}$ with extrema $x, y$ and orientation from $x$ to $y$. Let $z_{1}, z_{2} \in \mathbb{R}$ such that $x=\pi\left(z_{1}\right)$ and $z_{2}>z_{1}$ is the minimal element of $\mathbb{R}$ such that $y=\pi\left(z_{2}\right)$. If the orientation 
of the interval $I$ is the anticlockwise one then we define for a function $f: \mathcal{S}^{1} \rightarrow \mathbb{R}$ the integration over the oriented interval as

$$
\int_{I} f(s) d s:=\int_{z_{1}}^{z_{2}} f(z) d z .
$$

If $\bar{I}$ is an interval of $\mathcal{S}^{1}$ having the same support as $I$ but opposite orientation then we define

$$
\int_{\bar{I}} f(s) d s=-\int_{I} f(s) d s .
$$

The diffusion process (3.1) on $\mathcal{S}^{1}$ may be non-reversible. The condition of reversibility (see for example Bakry et al., 2014; Colangeli et al., 2011) is $s(x)=\nabla G(x)$ where $G$ is a function defined on $\mathcal{S}^{1}$ i.e. a periodic function of period 1. This condition is equivalent to having (recall definitions $(3.5))$

$$
\int_{\mathcal{S}^{1}} s(x) d x=0
$$

In particular the function $S$ defined in (3.5) can be interpreted as a function on the ring $\mathcal{S}^{1}$ just as in the reversible case. In the reversible case the stationarity condition becomes $J(\mu)=0$ and the invariant measure coincides with (3.7). In the non-reversible case we have a more complicated solution. We will show that in this case the invariant measure can be naturally represented by a continuous version of the combinatorial construction illustrated in Section 2. We will then generalize this representation to arbitrary metric graphs.

3.1. Scaling limit. We discuss informally the diffusive scaling limit of a random walker. This is done to obtain the invariant measure of a diffusion process as the scaling limit of the invariant measure of the discrete walker. The computation for the scaling limit will be short and informal (but it could easily be turned into a rigorous one): once we have obtained the limiting form of the measure we can prove directly that this is the correct one. The aim of the computation is to show that the basic structure of the combinatorial representation of Section 2 is preserved in the limit, which thus yields a continuous version of the construction. This fact is shown in particular on a one-dimensional ring.

Since we want a diffusive scaling limit we need to consider reversible random walks. In particular we consider the most general reversible nearest neighbor random walk on the discrete circle with $N$ sites that we consider embedded into $\mathcal{S}^{1}$ with mesh $N^{-1}$. According to Andreucci et al. (2019) this is determined by a weight function $\alpha_{N}: V \rightarrow \mathbb{R}^{+}$and a weight function $Q_{N}: E \rightarrow \mathbb{R}^{+}$such that $Q_{N}(x, y)=Q_{N}(y, x)$. A random walk is reversible if and only if the jump rates are fixed by

$$
r_{N}(x, y):=\alpha_{N}(x) Q_{N}(x, y), \quad y=x \pm \frac{1}{N} .
$$

We consider the case when the weight function $\alpha_{N}$ is obtained by the discretization of a $C^{2}$ function $\alpha: \mathcal{S}^{1} \rightarrow \mathbb{R}^{+}$by fixing $\alpha_{N}(x):=\alpha(x)$ when $x \in V$. The weight function $Q_{N}$ is likewise obtained by the discretization of a $C^{2}$-function $Q: \mathcal{S}^{1} \rightarrow \mathbb{R}^{+}$by fixing $Q_{N}(x, y):=Q\left(\frac{x+y}{2}\right)$. We assume that $\alpha$ and $Q$ are strictly positive.

We allow perturbation of rates by the switching on of a weak external field. The scaling behavior stays again diffusive. We consider a $C^{1}$-vector field $F$ on $\mathcal{S}^{1}$. This is a $C^{1}$-periodic function $F: \mathbb{R} \rightarrow \mathbb{R}$. The discretized version is a discrete vector field $F_{N}: E \rightarrow \mathbb{R}$ defined by

$$
F_{N}(e):=\int_{e} F(x) d x, \quad e \in E
$$

where the integral in (3.12) is the integral on the oriented segment going from the tail of the directed edge $e \in E$ to its head. By definition we have $F_{N}\left(x, x+\frac{1}{N}\right)=-F_{N}\left(x+\frac{1}{N}, x\right)$. 
The perturbed rates (Andreucci et al., 2019) are defined by

$$
r_{N}^{F}(x, y):=r_{N}(x, y) e^{F_{N}(x, y)} .
$$

We stress the difference between the two discretizations for $F_{N}$ and $Q_{N}$. We have indeed that $F_{N}(x, y)=O\left(N^{-1}\right)$ while instead $Q_{N}(x, y)=O(1)$.

According to the general discussion in Andreucci et al. (2019), when the lattice is of mesh $1 / N$ and the rates are rescaled by a factor of $N^{2}$ (diffusive rescaling) we obtain that the law of the random walk converges to the law of a diffusion process with a forward Kolmogorov equation (Gardiner, 2009) for the evolution of the probability measures given by

$$
\partial_{t} \mu_{t}(x)=-\partial_{x} J\left(\mu_{t}\right)=\partial_{x}\left[Q(x) \partial_{x}\left(\alpha(x) \mu_{t}(x)\right)\right]-2 \partial_{x}\left[\alpha(x) Q(x) F(x) \mu_{t}(x)\right] .
$$

Comparing (3.14) with (3.3) we obtain the relation between the macroscopic parametrization of the diffusion process, that is given by $(b, \sigma)$ and the microscopic one that is determined by $(\alpha, Q, F)$. We obtain

$$
\left\{\begin{array}{l}
\sigma=\sqrt{2 Q \alpha} \\
b=\alpha\left(2 Q F+\partial_{x} Q\right)
\end{array} .\right.
$$

Let us discuss the inverse transformations of (3.15). The first equation gives $\alpha=\frac{\sigma^{2}}{2 Q}$. If we insert this in the second one we get

$$
\frac{\partial_{x} Q}{2 Q}=s-F
$$

Since the left hand side of (3.16) is a total derivative, i.e. its integral on the circle vanishes, we have that if a triple $(Q, \alpha, F)$ satisfies (3.15) then we have

$$
\int_{\mathcal{S}^{1}} F(x) d x=\int_{\mathcal{S}^{1}} s(x) d x .
$$

Once an external field $F$ satisfying (3.17) has been fixed then the weights $Q, \alpha$ are uniquely determined as

$$
\left\{\begin{array}{l}
Q(x)=c e^{[S+2 V](x)}, \\
\alpha(x)=\frac{\sigma^{2}(x)}{2 c} e^{-[S+2 V](x)},
\end{array}\right.
$$

where $c$ is an arbitrary positive constant and

$$
[S+2 V](x):=\int_{I^{ \pm}\left[x^{*}, x\right]} 2(s(z)-F(z)) d z,
$$

where $x^{*} \in \mathcal{S}^{1}$ is an arbitrary point and the sign \pm can be chosen arbitrarily since the result does not change due to (3.17). In formulas (3.18) and (3.19) we use the notation $[S+2 V]$ in place of $S+2 V$ since in general the single functions $S$ and $V$ will not be well defined functions on the ring. We use the symbol $V$ following some classic notation for the potential even if this is the same symbol denoting the set of vertices. Since they are very different objects there will be no risk of confusion.

Remark 3.1. The above computations are independent of the boundary conditions and they hold also in the case that the lattice is embedded on an interval with suitably boundary conditions. In this case the external field $F$ can be fixed arbitrarily since for any external field the equation (3.16) can be solved in $Q$ on an interval. Given an arbitrary external field $F$, if we call $V(x):=-\int_{x^{*}}^{x} F(y) d y$ and $S(x):=2 \int_{x^{*}}^{x} s(y) d y$ where $x^{*}$ is an arbitrary point of the interval, then the weights $Q, \alpha$ are uniquely determined up to the choice of an arbitrary positive constant $c$ as in (3.18). 
3.1.1. Scaling limit of the invariant measure on the ring. Recall that the discrete walker is evolving on a discrete ring of mesh $1 / N$ embedded into $\mathcal{S}^{1}$ and that we draw the lattice as a ring on which the anticlockwise orientation corresponds to move from site $x$ to site $x+\frac{1}{N}$.

We will use in the discrete setting the following notation similar to the continuous one. Given $x \neq y \in V$, with the symbol $I^{+}[x, y]$ we mean the subgraph of $(V, E)$ containing the vertices and the directed edges that are visited by a walker moving from $x$ to $y$ anti-clockwise, $x$ and $y$ included. Likewise with the symbol $I^{-}[x, y]$ we mean the subgraph of $(V, E), x$ and $y$ included, containing the vertices and the directed edges that are visited by a walker moving from $x$ to $y$ clockwise. Note that according to our definition the vertices belonging to $I^{+}[x, y]$ and $I^{-}[y, x]$ are the same but they contain oppositely oriented edges.

We introduce the following notation

$$
R_{N}^{ \pm}(x, y):= \begin{cases}\prod_{e \in I^{ \pm}[x, y]} r_{N}^{F}(e) & y \neq x \\ 1 & y=x\end{cases}
$$

For the rates (3.13) we have that (3.20) becomes

$$
R_{N}^{ \pm}(x, y)=\alpha^{-1}(y)\left(\prod_{z \in I^{ \pm}[x, y]} \alpha(z)\right)\left(\prod_{e \in I^{ \pm}[x, y]} Q(e)\right) e^{\int_{I^{ \pm}[x, y]} F(z) d z} .
$$

Using the matrix tree Theorem discussed in Section 2 we can write the invariant measure for the walker on the ring of mesh $1 / N$ as

$$
\mu^{N}(x)=\frac{1}{Z_{N}} \sum_{y \in V} R_{N}^{+}\left(y+\frac{1}{N}, x\right) R_{N}^{-}(y, x),
$$

where $Z_{N}$ is a normalization factor. Using (3.21), for any $y$ we have

$$
R_{N}^{+}\left(y+\frac{1}{N}, x\right) R_{N}^{-}(y, x)=\left(\prod_{z \in V} \alpha(z)\right)\left(\prod_{e \in E} \sqrt{Q(e)}\right) \frac{e^{\int_{I^{+}\left[y+\frac{1}{N}, x\right]} F(z) d z} e^{\int_{I^{-}[y, x]} F(z) d z}}{\alpha(x) Q\left(y, y+\frac{1}{N}\right)} .
$$

The product is over directed edges and for this reason there is a square root. Using the above formula we obtain therefore that (3.22) converges to

$$
\mu(x)=\frac{1}{Z^{\prime} \alpha(x)} \int_{\mathcal{S}^{1}} d y \frac{e^{\int_{I^{+}(y, x)} F(z) d z} e^{\int_{I^{-}[y, x]} F(z) d z}}{Q(y)},
$$

where $Z^{\prime}$ is a suitable normalization constant.

Formula (3.23) is written in terms of the parameters of the microscopic walker. Using relation (3.15) and its inverse we can show that for any triple $(\alpha, Q, F)$ corresponding to a given $(b, \sigma)$ formula (3.23) coincides with

$$
\mu(x)=\frac{1}{Z \sigma^{2}(x)} \int_{\mathcal{S}^{1}} d y e^{\left[\int_{I^{+}[y, x]} s(z) d z+\int_{I^{-}[y, x]} s(z) d z\right]},
$$

where $Z$ is a normalization constant. Indeed according to (3.16) we have

$$
e^{\int_{I^{ \pm}(y, x)} F(z) d z}=e^{\int_{I^{ \pm}(y, x)} s(z) d z-\int_{I^{ \pm}(y, x)} \frac{\partial z Q}{2 Q}(z) d z}=e^{\int_{I^{ \pm}(y, x)} s(z) d z}\left(\frac{Q(y)}{Q(x)}\right)^{\frac{1}{2}}
$$

and (3.24) is obtained.

The geometric interpretation of (3.24) is very clear. Fix a point $x \in \mathcal{S}^{1}$ where we want to compute the density of the invariant measure. The density is then obtained by summing some weights over all possible continuous arborescences of $\mathcal{S}^{1}$ directed toward $x$. A continuous directed arborescense is obtained by cutting $\mathcal{S}^{1}$ on a point $y \in \mathcal{S}^{1}$ and by orienting the segments toward $x$ (see Figure 3.1 and Section 4 for precise definitions). The weight of the oriented continuos arborescence is obtained 


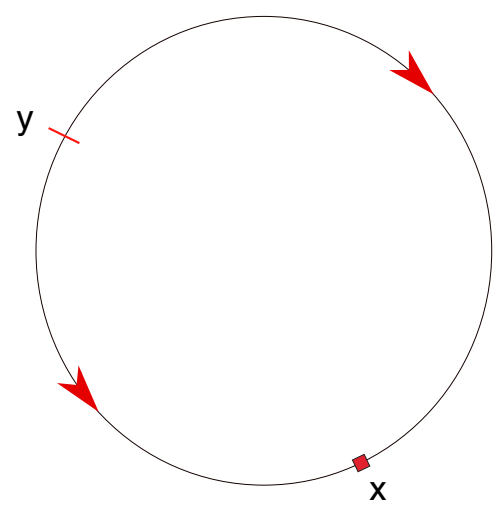

Figure 3.1. A continuous arborescence oriented toward the point $x$ obtained cutting $\mathcal{S}^{1}$ on the point $y$ and orienting $I^{+}[y, x]$ and $I^{-}[y, x]$ from $y$ to $x$.

by multiplying by a factor of $\sigma^{-2}(x)$ the exponential of the sum of integrals of the form $\int s$ over the oriented segments. The construction is therefore a direct continuous generalization of the discrete construction except for the appearance of the factor $\sigma^{-2}(x)$, related to the position of the root that it is not present in the discrete case.

Remark 3.2. Formula (3.24) can be also be written as

$$
\mu(x)=\frac{1}{Z \sigma^{2}(x)} \int_{x}^{x+1} e^{S(x)-S(y)} d y,
$$

that is a generalization of formula (2.3) in Faggionato and Gabrielli (2012) which is particularly useful in the small noise limit. We underline that formula (3.25) holds in the general case and not only under the condition (3.10).

3.2. Direct proof. We computed shortly the scaling limit of the Markov chain matrix tree Theorem. We give now a direct proof that the obtained formula is the invariant measure of the limiting diffusion process. This is an elementary fact that will however be used in the following and similar computations will be relevant in the more general case. Once again we stress that the importance of formula (3.24) is in the geometric interpretation.

Lemma 3.3. The unique invariant measure of the diffusion process (3.1) on $\mathcal{S}^{1}$ is given by (3.24).

Proof: Uniqueness is classic, see for example Bakry et al. (2014). We prove invariance by a direct computation. A key property is that when $y \neq x$ we have

$$
\partial_{x}\left[\int_{I^{+}[y, x]} s(z) d z\right]=\partial_{x}\left[\int_{I^{-}[y, x]} s(z) d z\right]=s(x) .
$$

By this computation we deduce that if we call $\psi(y, x)$ the integrand in (3.24), then this function is differentiable when $x \neq y$ and we have

$$
\partial_{x} \psi(y, x)=2 s(x) \psi(y, x), \quad x \neq y .
$$

Moreover $\psi$ has a discontinuity at $y=x$ given by

$$
\Delta \psi(x, x)=\lim _{\epsilon \downarrow 0}(\psi(x-\epsilon, x)-\psi(x+\epsilon, x))=e^{-\int_{\mathcal{S}^{1}} s(z) d z}-e^{\int_{\mathcal{S}^{1}} s(z) d z} .
$$




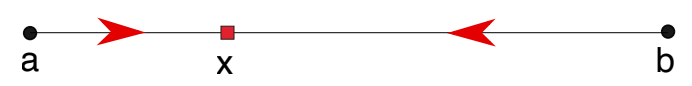

FiguRE 3.2. The continuous arborescence in the case of the interval. There are no cuts to be done and the orientation is drawn with red arrows.

Recall that as usual in the above formula we considered $\mathcal{S}^{1}$ anticlockwise oriented. Note that the jump at the discontinuous points does not depend on $x$ and therefore we call just $\Delta \psi$ the right hand side of (3.26). We can proceed as follows

$$
\begin{aligned}
& \partial_{x}\left(\int_{\mathcal{S}^{1}} \psi(y, x) d y\right) \\
& =\partial_{x}\left(\int_{I^{+}[x+\epsilon, x-\epsilon]} \psi(y, x) d y\right)+\partial_{x}\left(\int_{I^{+}[x-\epsilon, x+\epsilon]} \psi(y, x) d y\right) \\
& =2 s(x)\left(\int_{I^{+}[x+\epsilon, x-\epsilon]} \psi(y, x) d y\right)+\psi(x-\epsilon, x)-\psi(x+\epsilon, x) \\
& +\partial_{x}\left(\int_{I^{+}[x-\epsilon, x+\epsilon]} \psi(y, x) d y\right) .
\end{aligned}
$$

The last term in the above chain can be shown to be negligible in the limit $\epsilon \downarrow 0$ so that we deduce taking this limit on the right hand side of the above computation

$$
\partial_{x}\left(\int_{\mathcal{S}^{1}} \psi(y, x) d y\right)=2 s(x)\left(\int_{\mathcal{S}^{1}} \psi(y, x) d y\right)+\Delta \psi
$$

Inserting these computations into (3.3) we obtain

$$
J(\mu)=-\frac{\Delta \psi}{2 Z}
$$

which is constant and represents the typical value of the current across the ring. We have therefore that (3.4) is satisfied and this implies that (3.24) is the unique invariant measure of (3.1) on $\mathcal{S}^{1}$. This is because it is naturally periodic, it is not negative, normalized to one and satisfies (3.2).

Remark 3.4. In the case of an interval $[a, b]$ there are no cycles and once the point $x$ where to compute the density is fixed there are no cuts to be done. The oriented arborescence is obtained by orienting the intervals $[a, x]$ and $[b, x]$ towards $x$, see Figure 3.2. We get from the scaling limit

$$
\mu(x)=\frac{1}{Z \sigma^{2}(x)} e^{\left[\int_{a}^{x} s(z) d z+\int_{b}^{x} s(z) d z\right]} .
$$

Formula (3.7) coincides with (3.28) since

$$
\int_{a}^{x} s(z) d z+\int_{b}^{x} s(z) d z=2 \int_{x^{*}}^{x} s(z) d z+c
$$

with $c$ a suitable additive constant. 


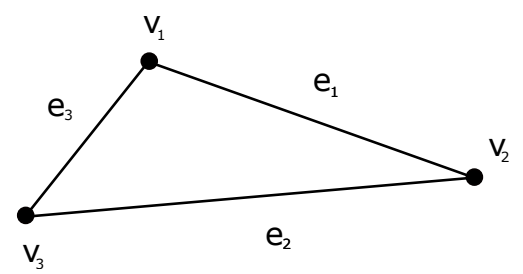

FigURE 4.3. A very simple example of metric graph: a triangle obtained suitably gluing the 3 metric edges $\mathbf{e}_{i}, i=1,2,3$ to 3 vertices $v_{i}, i=1,2,3$.

\section{Metric graphs and metric arborescences}

We give a quick and informal description of a family of metric spaces called metric graphs. We refer for more details for example to Mugnolo (2014) or Burago et al. (2001) Section 3.2.2.

We describe a finite metric graph $\mathcal{G}=(V, \mathbf{E})$. The set of vertices $V$ is a finite set and each element $v \in V$ is just a single point called a vertex. The set of metric edges $\mathbf{E}$ is also a finite set containing $|\mathbf{E}|$ metric edges. An element $\mathbf{e} \in \mathbf{E}$ is identified with an open interval $\mathbf{e}=\left(0, \ell_{\mathbf{e}}\right), \ell_{\mathbf{e}} \in(0, \infty)$. A metric graph is a metric space obtained by suitably gluing the intervals associated with the edges to the vertices in $V$. More precisely, when we glue the edge $\mathbf{e} \in \mathbf{E}$ to the vertex $v \in V$, this means that we identify one of the two extrema of e with $v$. Note that, as explained in the following, the metric edges have an intrinsic orientation (the one corresponding to increasing coordinates) and the gluing can be done in two different ways, namely by identifying $v$ with the endpoint corresponding to either the coordinate 0 or to the coordinate $\ell_{\mathbf{e}}$. If the orientation is disregarded the two identifications turn out to be equivalent. We allow also for a third possibility, corresponding to the case in which $v$ is identified with both endpoints of e, thus obtaining a ring of length $\ell_{e}$ with the marked point $v$. When a vertex is identified with one endpoint of a metric edge we say that the edge is incident to the vertex. Every endpoint of each edge must be identified with exactly one vertex and therefore we do not allow to have endpoints of edges not identified with any vertex (i.e. the procedure ends after exactly $2|\mathbf{E}|$ identifications). Moreover me may have multiple edges. This means that we may have two or more metric edges that have their two endpoints identified with the same pair of vertices. If one endpoint of the metric edge $\mathbf{e}$ and one endpoint of the metric edge $\mathbf{e}^{\prime}$ are identified with the same vertex $v$ then automatically the two identified endpoints of the two metric edges are identified with each other.

The metric graph is obtained starting from the collection of vertices and metric edges and performing a finite number of identifications of vertices and endpoints according to the above rules.

One of the simplest possible examples is illustrated in Figure 4.3, where a triangle is obtained gluing the 3 metric edges $\mathbf{e}_{i}, i=1,2,3$, to the 3 vertices $v_{i}, i=1,2,3$. In particular the endpoints of $\mathbf{e}_{1}$ are identified with $v_{1}$ and $v_{2}$; the endpoints of $\mathbf{e}_{2}$ are identified with $v_{2}$ and $v_{3}$ and the endpoints of $\mathbf{e}_{3}$ are identified with $v_{3}$ and $v_{1}$.

Each metric edge is itself a metric space but it is a very special one, indeed a one dimensional segment. For this reason after identifications we have a well defined notion of distance between different points of the metric graph. The distance between two points on $\mathcal{G}$ is the length of the minimal path moving along the edges and going from one endpoint of one edge to an endpoint of another edge if both are identified with the same vertex. We underline that this notion of distance is independent of the orientation of the edges.

If we disregard orientation of the metric edges and consider them just as segments of length $\ell_{\mathbf{e}}$ we can identify the metric structure of the metric graph by giving just its combinatorial arrangement and the lengths. More precisely given $v \in V$ we denote by $A^{+}(v), A^{-}(v) \subset \mathbf{E}$ the metric edges that are respectively exiting from the vertex $v$ and entering into the vertex $v$. Moreover we define 
$A(v)=A^{+}(v) \cup A^{-}(v)$. Given a metric graph $(V, \mathbf{E})$ we construct the corresponding unoriented graph $(V, \mathcal{E})$ defined as follows. The set of vertices is again $V$ and given $v, w \in V$ we have that there is a number of unoriented edges $\{v, w\} \in \mathcal{E}$ equal to $|A(v) \cap A(w)|$. We have moreover a number of loops $\{v, v\}$ equal to $\left|A^{+}(v) \cap A^{-}(v)\right|$. The unoriented graph is weighted and the edge $\{v, w\} \in \mathcal{E}$ corresponding to $\mathbf{e} \in \mathbf{E}$ has a weight given by $\ell_{\mathbf{e}}$.

The metric graph $(V, \mathbf{E})$ is connected if $(V, \mathcal{E})$ is connected and it is a metric tree if $(V, \mathcal{E})$ is a tree.

Formally this can be equivalently formulated as follows. A path from $x \in(V, \mathbf{E})$ to $y \in(V, \mathbf{E})$ is a continuous map $\psi:[a, b] \rightarrow(V, \mathbf{E})$, where $a<b$ are some given real parameters, and such that $\psi(a)=x$ and $\psi(b)=y$. When we write $x, y \in(V, \mathbf{E})$ we mean that $x, y$ are generic elements of the metric graph i.e. they can be points on an edge or vertices. We underline that a path is just a continuous map from an interval to the metric space $(V, \mathbf{E})$ so that in general has not to satisfy any relation with orientation. A metric graph is connected if for any pair $x, y \in(V, \mathbf{E})$ there exists a path from $x$ to $y$. A metric graph is called a metric tree if for any two points $x, y \in(V, \mathbf{E})$ there is an unique injective path, up to reparametrizations, going from $x$ to $y$.

In order to draw pictures, it is very useful to consider the graph embedded into $\mathbb{R}^{d}$ but the specific embedding is irrelevant. The vertices are therefore points of $\mathbb{R}^{d}$. The edges are disjoint and non-self intersecting regular curves of length $\ell_{\mathbf{e}}$. Every edge connects two vertices of $V$ or just one in the case of loops. An edge $\mathbf{e} \in \mathbf{E}$ is parametrically described by the corresponding interval $\left(0, \ell_{\mathbf{e}}\right)$, with $\ell_{\mathbf{e}} \in(0, \infty)$, and a $C^{1} \operatorname{map} \phi_{\mathbf{e}}:\left(0, \ell_{\mathbf{e}}\right) \rightarrow \mathbb{R}^{d}$ such that $\left|\phi_{\mathbf{e}}^{\prime}\right|(x)=1$ for any $x \in\left(0, \ell_{\mathbf{e}}\right)$. The vertices connected by the edge $\mathbf{e}$ are recovered by $\lim _{x \downarrow 0} \phi_{\mathbf{e}}(x)$ and $\lim _{x \uparrow \ell_{\mathbf{e}}} \phi_{\mathbf{e}}(x)$.

We allow for the possibility of cutting some of the edges of the metric graph. Given $\mathbf{e} \in \mathbf{E}$ and $x \in \mathbf{e}$ when we cut the metric graph at $x$ we remove the point $x$ from the metric graph $(V, \mathbf{E})$. The new metric graph obtained after the cut is as follows. The structure remains unchanged for all the edges that do not contain the cutting point while the edge e containing $x$ is removed and substituted by two different edges $\mathbf{e}_{1}=(0, x)$ and $\mathbf{e}_{2}=\left(0, \ell_{\mathbf{e}}-x\right)$. Henceforth, with a slight abuse of notation, we call $x \in \mathbf{e}$ both the point on the edge and its coordinate on the corresponding interval $\left(0, \ell_{\mathbf{e}}\right)$. The endpoint 0 of the first edge is identified with the same vertex of the endpoint 0 of $\mathbf{e}$. The endpoint $\ell_{\mathbf{e}}-x$ of the second edge is identified with the same vertex of the endpoint $\ell_{\mathbf{e}}$ of $\mathbf{e}$. Finally we add two new vertices $v_{1}, v_{2}$ and identify $v_{1}$ with the endpoint $x$ of $\mathbf{e}_{1}$ and $v_{2}$ with the endpoint 0 of $\mathbf{e}_{2}$. See Figure 4.4 for an illustrative example.

Note that before the cut the points of e with coordinates $x-\epsilon$ and $x+\epsilon$ for $\epsilon$ small enough are at distance $2 \epsilon$ while this is no more the case after the cut.

Every edge of a metric graph is naturally oriented according to the increasing direction of the coordinates. The opposite orientation corresponds to the decreasing direction. There are just two possible orientations for each edge. An orientation of a metric graph is simply a choice between the two possible orientations for each edge. An orientation is represented by drawing an arrow on each edge. The canonical orientation of a metric graph is the one on which each edge is increasingly oriented. Given a vertex $v \in V$, according to our notation, $A^{+}(v)$ and $A^{-}(v)$ are the sets of edges incident to $v$ that are respectively canonically oriented exiting from $v$ or entering into $v$. A path $\psi:[a, b] \rightarrow(V, \mathbf{E})$ is compatible with the orientations of the edges if when restricted to each edge the map $\psi$ is increasing or decreasing depending if the edge is increasingly or decreasingly oriented.

A metric arborescence oriented toward $x \in \mathbf{E}$ is a metric graph that is a metric tree and moreover all the edges are oriented toward the root $x$. This means that given any $y \in(V, \mathbf{E})$ there exists an unique injective path from $y$ to $x$ and the path is compatible with the orientations. This essentially means that it is possible to reach the root $x$ starting from any other point $y$ and moving on the graph following the edges according to their orientation. Note that on a metric arborescence the edge containing the root is divided into two parts oppositely oriented. 


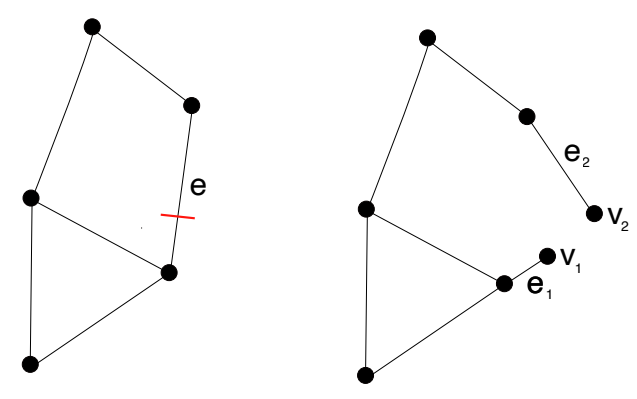

FiguRE 4.4. A metric graph on the left with a special point marked with a red cross. We cut the edge at the cross and obtain a new metric graph. The metric edge $\mathbf{e}$ is transformed into two metric edges $\mathbf{e}_{i}, i=1,2$ and two new vertices $v_{i}, i=1,2$ are created.

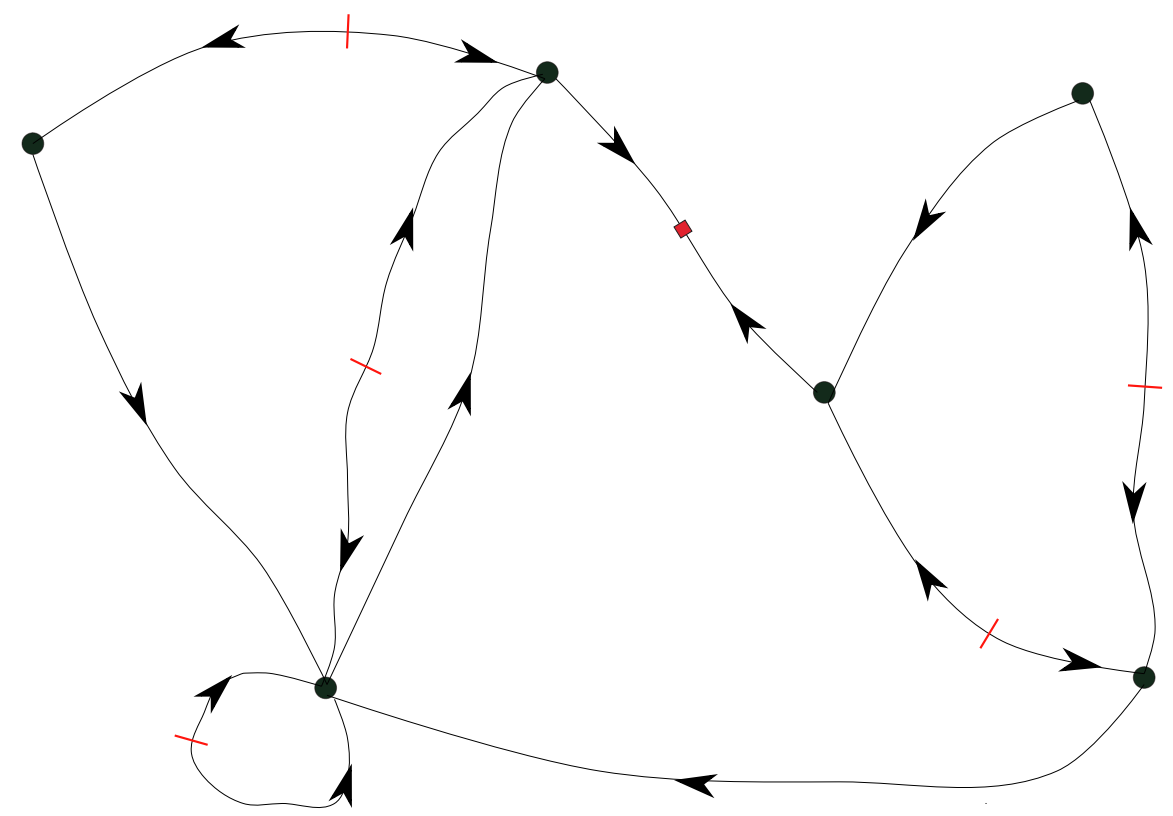

FiguRE 4.5. A metric graph embedded into $\mathbb{R}^{d}$ and an associated oriented arborescence obtained cutting the edges on the green slices. The root of the arborescence is drawn as a red dot. Note that loops and multiple edges are allowed.

Given a metric graph $\mathcal{G}$ we can obtain a metric arborescence by cutting some of the edges and suitably orienting the edges, see Figure 4.5 for an example and the following Section 6 for a more detailed description.

\section{Diffusions on metric graphs}

A walker is moving randomly on $\mathcal{G}=(V, \mathbf{E})$ according to the following mechanism. For each edge $\mathbf{e} \in \mathbf{E}$ we fix coefficients $\left(b_{\mathbf{e}}, \sigma_{\mathbf{e}}\right)$ defined on the intervals $\left(0, \ell_{\mathbf{e}}\right), \ell_{\mathbf{e}} \in \mathbb{R}$, and again for simplicity we require $b_{\mathbf{e}} \in C^{1}$ and $\sigma_{\mathbf{e}} \in C^{2}$. The coefficients can be extended continuously on $\left[0, \ell_{\mathbf{e}}\right]$ and we require 
$\min _{x \in\left[0, \ell_{\mathbf{e}}\right]} \sigma_{\mathbf{e}}(x)>0$. When the walker is on the edge $\mathbf{e}$ then the coordinate of the walker on $\left(0, \ell_{\mathbf{e}}\right)$ evolves as a diffusion process $X_{\mathbf{e}}(t)$ satisfying the equation (3.1) with coefficients $\left(b_{\mathbf{e}}, \sigma_{\mathbf{e}}\right)$. When the walker reaches a vertex $v \in V$ then she can spend some time there and then the new edge on which she continues the evolution is chosen randomly according to a given probability distribution on $A(v)$. The precise formulation of the dynamics can be formalized using the excursions of the diffusion processes, see for example Barlow et al. (1989); Walsh (1978). In the following we will use an alternative approach to defining the dynamics via the the corresponding generator.

We present a formal definition of the diffusion processes on a metric graph by describing the structure of the generator. We state the basic facts and refer to Freidlin and Sheu (2000); Freidlin and Wentzell $(1993,1994)$ for a general discussion. We give a short and essential summary of the features of the possible dynamics, all of them depend on the behavior of the walker at the nodes.

All the possible Feller processes with continuous sample path and such that inside each edge $\mathbf{e} \in \mathbf{E}$ the random dynamics coincides with a diffusion with parameters $\left(b_{\mathbf{e}}, \sigma_{\mathbf{e}}\right)$ are determined by a family of nonnegative parameters $\alpha$ 's. In particular we have a family of parameters associated to the vertices $\left(\alpha_{v}\right)_{v \in V}$ and a family of parameters associated to the pairs $(v, \mathbf{e})$ such that $v \in V$ and $\mathbf{e} \in A(v)$. We denote them as $\left(\alpha_{v, \mathbf{e}}\right)_{v \in V, \mathbf{e} \in A(v)}$. The parameters must satisfy the constraints

$$
\alpha_{v}+\sum_{\mathbf{e} \in A(v)} \alpha_{v, \mathbf{e}}>0, \quad \forall v \in V .
$$

Once a collection of parameters is fixed then the process is defined by its generator $\mathcal{A}$ that is a linear operator on a suitable subset of $C(\mathcal{G})$, which is the set of continuous function on the metric graph $\mathcal{G}$. To describe the form of the generator we need some notation. To avoid problems of irreducibility we consider always metric graphs that are connected and we will assume that each coefficient $\alpha_{v, \mathbf{e}}$ is strictly positive:

$$
\alpha_{v, \mathbf{e}}>0, \quad \forall v \in V \text { and } \forall \mathbf{e} \in A(v) .
$$

A function $f \in C(\mathcal{G})$ is determined by a family of continuous functions $f_{\mathbf{e}}:\left(0, \ell_{\mathbf{e}}\right) \rightarrow \mathbb{R}$. The value of the function $f$ on a point $x \in \mathbf{e}$ is given by $f(x):=f_{\mathbf{e}}(x)$. Here again with abuse of notation we call $x \in \mathbf{e}$ both the point and its coordinate in $\left(0, \ell_{\mathbf{e}}\right)$. In order that $f \in C(\mathcal{G})$ we need to impose the condition that given any $v \in V$ there is a real number $f(v) \in \mathbb{R}$ (the value of the function at the vertex $v$ ) such that

$$
f(v)=\lim _{x \uparrow \ell_{\mathbf{e}}} f_{\mathbf{e}}(x)=\lim _{x \downarrow 0} f_{\mathbf{e}^{\prime}}(x), \quad \forall \mathbf{e} \in A^{-}(v), \mathbf{e}^{\prime} \in A^{+}(v) .
$$

Given $v \in V$, e $\in A(v)$ and $f \in C(\mathcal{G})$ we define $D_{\mathbf{e}} f(v)$ the exiting derivative of $f$ at $v$ along $\mathbf{e}$ as

$$
D_{\mathbf{e}} f(v):= \begin{cases}\lim _{x \downarrow 0} \frac{f_{\mathbf{e}}(x)-f(v)}{x}, & \mathbf{e} \in A^{+}(v), \\ \lim _{x \uparrow \ell_{\mathbf{e}}} \frac{f_{\mathbf{e}}(x)-f(v)}{\ell_{\mathbf{e}}-x}, & \mathbf{e} \in A^{-}(v),\end{cases}
$$

when the limits exist.

We define now the generator $\mathcal{A}$ of our dynamics.

Definition 5.1 (Generator of the dynamics). Consider a function $f \in C(\mathcal{G})$ with $f_{\mathbf{e}} \in C^{2}\left(0, \ell_{\mathbf{e}}\right)$. For an $x \in \mathbf{e} \in \mathbf{E}$ we define

$$
[\mathcal{A} f](x):=L_{\mathbf{e}} f_{\mathbf{e}}(x),
$$

where $L_{\mathbf{e}}$ is the generator of the diffusion process with parameters $\left(b_{\mathbf{e}}, \sigma_{\mathbf{e}}\right)$, i.e.

$$
L_{\mathbf{e}} f_{\mathbf{e}}(x):=\frac{1}{2} \sigma_{\mathbf{e}}^{2}(x) \partial_{x}^{2} f_{\mathbf{e}}(x)+b_{\mathbf{e}}(x) \partial_{x} f_{\mathbf{e}}(x), \quad x \in\left(0, \ell_{\mathbf{e}}\right)
$$

The domain of definition $\mathcal{D}(\mathcal{A}) \subseteq C(\mathcal{G})$ of the operator $\mathcal{A}$ is the set of functions for which all the derivatives exist and such that $\mathcal{A} f \in C(\mathcal{G})$. In particular we have

$$
\mathcal{A} f(v):=\lim _{x \uparrow \ell_{\mathbf{e}}} L_{\mathbf{e}} f_{\mathbf{e}}(x)=\lim _{x \downarrow 0} L_{\mathbf{e}} f_{\mathbf{e}^{\prime}}(x), \quad \forall \mathbf{e} \in A^{-}(v), \mathbf{e}^{\prime} \in A^{+}(v) .
$$


Finally on the nodes the following relation has to be satisfied

$$
\alpha_{v}[\mathcal{A} f](v)=\sum_{\mathbf{e} \in A(v)} \alpha_{v, \mathbf{e}} D_{\mathbf{e}} f(v), \quad v \in V
$$

Note that in the case $\alpha_{v}=0$ then relation (5.2) does not involve $[\mathcal{A} f](v)$.

For the above definition 5.1 and the following Theorem 5.2 we refer to Freidlin and Sheu (2000), Freidlin and Wentzell (1993), Freidlin and Wentzell (1994). In particular for a result that classifies all the possible diffusions on metric graphs and a clear discussion of the form of the generator we refer to Section 3 of Freidlin and Wentzell (1993); next theorem is Theorem 3.1 there.

Theorem 5.2. The operator $\mathcal{A}$ as defined in Definition 5.1 is the infinitesimal generator of a strongly continuous semigroup of operators on $C(\mathcal{G})$ corresponding to a conservative Markov process on $\mathcal{G}$ with continuous paths. The following statements hold:

- Before the process leaves an edge $\mathbf{e}$ it evolves like a diffusion process with generator $L_{\mathbf{e}}$ as in (5.1)

- If $\alpha_{v}=0$ then the process spends almost surely zero time at the vertex $v \in V$

- If $\alpha_{v}=0$ for any $v \in V$ then the distribution of the process at all given time has a density with respect to a measure that is zero on all the vertices $v \in V$

Conversely, if $X(t)$ is a Feller conservative Markov process on $\mathcal{G}$ which coincides, before leaving $\mathbf{e}$, with a diffusion having generator (5.1) then its infinitesimal generator coincides with $\mathcal{A}$ in Definition 5.1 for a suitable choice of the parameters. Moreover if the process $X$ spends almost surely zero time at $v \in V$ then necessarily $\alpha_{v}=0$.

We discuss now the invariant measures of diffusion processes on metric graphs. The conditions that we obtain can be easily described in terms of a divergence free condition for the probability currents (3.3) on the edges. Fix an arbitrary reference orientation for each edge that for us will be always the canonical one. For $v \in V, \mathbf{e} \in A(v)$ and a collection of functions $\left(g_{\mathbf{e}}(x), x \in\left(0, \ell_{\mathbf{e}}\right)\right)_{\mathbf{e} \in \mathbf{E}}$ we denote by

$$
g_{\mathbf{e}}(v):=\lim _{y \in \mathbf{e}, y \rightarrow v} g_{\mathbf{e}}(y) .
$$

The invariant measures $\mu$ for this class of processes contain atomic components on the vertices and are absolutely continuous with respect to the Lebesgue measure on the edges. A measure of this type is denoted by

$$
\mu=\left\{\left(\mu_{v}\right)_{v \in V},\left(\mu_{\mathbf{e}}(x) d x\right)_{\mathbf{e} \in \mathbf{E}}\right\} .
$$

The number $\mu_{v}$ is the weight of the atomic component on the vertex $v \in V$ while $\mu_{\mathbf{e}}(x) d x$ is the density of the absolutely continuous component on the edge $\mathbf{e} \in \mathbf{E}$. The normalization condition is

$$
\sum_{v \in V} \mu_{v}+\sum_{\mathbf{e} \in \mathbf{E}} \int_{0}^{\ell_{\mathbf{e}}} \mu_{\mathbf{e}}(x) d x=1 .
$$

Given a measure $\mu$ with $C^{1}$ densities, we define a probability current $J[\mu]=\left(J_{\mathbf{e}}[\mu]\right)_{\mathbf{e} \in \mathbf{E}}$ on the edges of $\mathcal{G}$ by

$$
J_{\mathbf{e}}[\mu](x):=-\frac{1}{2} \partial_{x}\left(\sigma_{\mathbf{e}}^{2}(x) \mu_{\mathbf{e}}(x)\right)+b(x) \mu_{\mathbf{e}}(x), \quad x \in\left(0, \ell_{\mathbf{e}}\right) .
$$

The following is a direct consequence of Theorem 5.2, see also Jehring (2009). Note that in the following lemma, condition (3) is separated into $(3 A)$ and $(3 B)$; this splitting will be relevant in the following. When we say condition (3) we mean that both $(3 A)$ and $(3 B)$ are satisfied.

Lemma 5.3. Consider a diffusion process on a metric graph $\mathcal{G}$ having generator $\mathcal{A}$ as in Definition 5.1. Then the process has a unique invariant measure $\mu$ of the form (5.4) characterized by the following properties:

(1) The current $J_{\mathbf{e}}[\mu]$ is constant on each edge $\mathbf{e} \in \mathbf{E}$. 
(2) On each node $v \in V$ we have the divergence free condition

$$
\operatorname{div} J[\mu](v):=\sum_{\mathbf{e} \in A^{+}(x)} J_{\mathbf{e}}[\mu]-\sum_{\mathbf{e} \in A^{-}(x)} J_{\mathbf{e}}[\mu]=0 .
$$

(3) There exist some constants $\lambda_{v}>0$ such that

$$
\left\{\begin{array}{lll}
\frac{1}{2} \sigma_{\mathbf{e}}^{2}(v) \mu_{\mathbf{e}}(v)=\lambda_{v} \alpha_{v, \mathbf{e}} & v \in V, \mathbf{e} \in A(v), & (3 A), \\
\mu_{v}=\lambda_{v} \alpha_{v} & v \in V,
\end{array}\right.
$$

Furthermore the process is reversible if and only if conditions $(1)+(2)$ are replaced by the single condition

(1') $J_{\mathbf{e}}[\mu]=0$ on each edge $\mathbf{e} \in \mathbf{E}$.

Proof: Uniqueness follows using the same argument of Corollary 5.4.3 in Bogachev et al. (2015). The fact that the unique invariant measure is of the form (5.4) follows by Theorem 5.2. We show now that a probability measure $\mu$ satisfies the three conditions above if and only if

$$
\int_{\mathcal{G}}[\mathcal{A} f] d \mu=0, \quad \forall f \in \mathcal{D}(\mathcal{A}) .
$$

Consider a generic $f \in \mathcal{D}(\mathcal{A})$ and perform double integration by parts on each edge. We obtain the following

$$
\begin{aligned}
\int_{\mathcal{G}}[\mathcal{A} f] d \mu= & \sum_{v \in V}\left[\mu_{v}[\mathcal{A} f](v)-\sum_{\mathbf{e} \in A(v)} \frac{1}{2} \sigma_{\mathbf{e}}^{2}(v) \mu_{\mathbf{e}}(v) D_{\mathbf{e}} f(v)\right]+\sum_{v \in V} f(v) \operatorname{div} J[\mu](v) \\
& -\sum_{\mathbf{e} \in \mathbf{E}} \int_{0}^{\ell_{\mathbf{e}}} f_{\mathbf{e}}(x) \partial_{x} J_{\mathbf{e}}(\mu) d x .
\end{aligned}
$$

Condition (1) implies that each term of the third sum on the right hand side of the above equation is zero. Condition (2) implies the same for the second sum and condition (3) for the first one. We have therefore that if the conditions are satisfied then (5.8) holds. Conversely since the right hand side of the above equation has to be zero for each function $f \in \mathcal{D}(\mathcal{A})$ then each single term has to be identically zero and this implies the validity of the three conditions.

We briefly outline why $\mathcal{D}(\mathcal{A})$ is large enough in order to guarantee that (5.8) implies conditions (1), (2) and (3). Consider first the last term in the right hand side of the above computation. Observe that if we consider $f \in \mathcal{D}(\mathcal{A})$ and we keep $f$ fixed on all the edges apart one single edge e where we add a compactly supported function, we obtain again a function in the domain. Only one of the terms in the last sum in the right hand side of the above computation is changing and since the perturbation is an arbitrary $C^{2}$ function compactly supported inside the edge we deduce the validity of (1) for each edge e. We consider now the second sum on the right hand side of the above computation. We can construct an element of $\mathcal{D}(\mathcal{A})$ as follows. First we fix the values of $\left(D_{\mathbf{e}} f(v)\right)_{\mathbf{e} \in \mathbf{E}}^{v \in V}$. Once fixed arbitrarily these numbers we have that $(\mathcal{A} f(v))_{v \in V}$ are determined by conditions (5.2) (we are considering the case $\alpha_{v}>0$ for any $v$, the other cases can be handled similarly). By continuity of $\mathcal{A} f$ we have therefore that the numbers $\left(\partial_{x}^{2} f_{\mathbf{e}}(v)\right)_{\mathbf{e} \in \mathbf{E}}^{v \in V}$ are determined. Any collection of $C^{2}$ functions on the edges that satisfy these constraints determines an element of the domain. We can then perturb this function obtaining a new function that is again in the domain adding a $C^{2}$ function that is different from zero just on edges in $A(v)$, it is compactly supported in a neighborood of $v$ and has first and second derivative equal to zero at $v$. In this way the new function on the domain has the same value of the original function on each vertex different from $v$ while in $v$ we can have a new arbitrary value. This means that condition (2) must hold and each term in the second sum in the right hand side of the above computation is necessarily zero. Finally since we can fix arbitrarily the values $\left(D_{\mathbf{e}} f(v)\right)_{\mathbf{e} \in \mathbf{E}}^{v \in V}$ (recall that we are considering the case $\alpha_{v}>0$ 
for any $v$, the other cases can be handled similarly) we can fix them is such a way that just one is different from zero and this implies directly condition (3).

The reversibility is proved observing that, for all $f, h \in \mathcal{D}(\mathcal{A})$, we get

$$
\begin{aligned}
\int_{\mathcal{G}}[\mathcal{A} f] h d \mu-\int_{\mathcal{G}} f[\mathcal{A} h] d \mu & =\sum_{v \in V}\left[\mu_{v}[\mathcal{A} f](v)-\sum_{\mathbf{e} \in A(v)} \frac{1}{2} \sigma_{\mathbf{e}}^{2}(v) \mu_{\mathbf{e}}(v) D_{\mathbf{e}} f(v)\right] h(v) \\
& -\sum_{v \in V}\left[\mu_{v}[\mathcal{A} h](v)-\sum_{\mathbf{e} \in A(v)} \frac{1}{2} \sigma_{\mathbf{e}}^{2}(v) \mu_{\mathbf{e}}(v) D_{\mathbf{e}} h(v)\right] f(v) \\
& +\sum_{\mathbf{e} \in \mathbf{E}} \int_{0}^{\ell_{\mathbf{e}}}\left(\partial_{x} f_{\mathbf{e}}(x) h_{\mathbf{e}}(x)-f_{\mathbf{e}}(x) \partial_{x} h_{\mathbf{e}(x)}\right) J_{\mathbf{e}}(\mu) d x
\end{aligned}
$$

The first two terms in the right hand side of the above formula are zero by (3). The third term is zero by condition $\left(1^{\prime}\right)$. The converse statement is proved observing that the above equation has to be zero for any pair of functions $f, h \in \mathcal{D}(\mathcal{A})$. The argument for this is very similar to the one of the general case and we do not give details.

Remark 5.4. Condition (3) of Lemma 5.3 can be written as

$$
\frac{\sigma_{\mathbf{e}}^{2}(v) \mu_{\mathbf{e}}(v)}{2 \mu_{v}}=\frac{\alpha_{v, \mathbf{e}}}{\alpha_{v}}, \quad v \in V, \mathbf{e} \in A(v),
$$

for the vertices for which $\alpha_{v}>0$ and as

$$
\frac{\sigma_{\mathbf{e}}^{2}(v) \mu_{\mathbf{e}}(v)}{\sigma_{\mathbf{e}^{\prime}}^{2}(v) \mu_{\mathbf{e}^{\prime}}(v)}=\frac{\alpha_{v, \mathbf{e}}}{\alpha_{v, \mathbf{e}^{\prime}}}, \quad \mathbf{e}, \mathbf{e}^{\prime} \in A(v),
$$

for the vertices for which $\alpha_{v}=0$. Note that for a vertex $v$ with $\alpha_{v}>0$ we have that condition (5.9) implies condition (5.10).

In the next section we give an explicit construction of a probability measure satisfying the conditions of Lemma 5.3, thereby describing the unique invariant measure of the process.

\section{A combinatorial representation of the invariant measure}

We consider metric arborescences rooted at $x \in \mathcal{G}$ that we recall are oriented metric trees obtained cutting the original metric graph on a finite number of points and orienting all the edges toward the root (see Figure 4.5 for an example). Let us call $\mathcal{T}_{x}$ the collection of all the metric arborescences rooted at $x \in \mathbf{e} \in \mathbf{E}$.

Given $\tau \in \mathcal{T}_{x}$ we define the corresponding weight as

$$
R(\tau):=\frac{e^{\int_{\tau} s}}{\sigma_{\mathbf{e}(x)}^{2}(x)} \prod_{v \in V} \mathcal{W}_{v}(\tau) .
$$

The symbol $\int_{\tau} s$ denotes the sum of all the integrals along the edges of the metric graph according to the orientations of $\tau$. In particular if the edge e does not contain a cut and it is oriented in $\tau$ according to its natural orientation then the contribution of the edge is given by $\int_{0}^{\ell_{\mathbf{e}}} s_{\mathbf{e}}(x) d x$. If instead the edge is oriented in $\tau$ oppositely with respect to the natural orientation then the contribution is $-\int_{0}^{\ell_{\mathbf{e}}} s_{\mathbf{e}}(x) d x$. If the edge $\mathbf{e}$ contains a cut at the point $z \in\left(0, \ell_{\mathbf{e}}\right)$ then the contribution coming from this edge is given by $-\int_{0}^{z} s_{\mathbf{e}}(x) d x+\int_{z}^{\ell_{\mathbf{e}}} s_{\mathbf{e}}(x) d x$ since the orientations of the two branches of the edge are necessarily exiting from the cut-point. Recall that as before we use the notation $s_{\mathbf{e}}:=\frac{b_{\mathbf{e}}}{\sigma_{\mathbf{e}}^{2}}$. 
The weight $\mathcal{W}_{v}(\tau)$ for the vertex $v \in V$ and the arborescence $\tau$ is defined by

$$
\mathcal{W}_{v}(\tau):=\prod_{\mathbf{e} \in A(v)} W_{v}^{\theta_{v}(\mathbf{e}, \tau)}(\mathbf{e})
$$

In (6.2) we have $\theta_{v}(\mathbf{e}, \tau)= \pm$ depending on whether $\mathbf{e}$ is oriented in the arborescence $\tau$ in a neighborhood of $v$ exiting or entering respectively into $v$. For any pair $v \in V$ and $\mathbf{e} \in A(v)$ we therefore have two free parameters $W_{v}^{ \pm}(\mathbf{e})>0$.

We fix $W_{v}^{-}(\mathbf{e}):=1$ for any edge $\mathbf{e}$, since this corresponds to multiplying the weights $R$ by a constant.

The number of cuts which have to be performed in order to transform a metric graph $(V, \mathbf{E})$ into a metric tree is fixed and it is given by $|\mathbf{E}|-|V|+1$. We call this number the dimension of the cut space. In order to obtain a metric tree the cuts have to be made on different edges. More precisely, the exact procedure is the following. Associate to the metric graph $(V, \mathbf{E})$ the unoriented graph $(V, \mathcal{E})$ as discussed in Section 4. According to our definitions, loops and multiple edges are allowed. By construction we have $|\mathbf{E}|=|\mathcal{E}|$ and there is a natural correspondence between metric edges and unoriented edges. Consider a spanning tree $(V, T)$ of $(V, \mathcal{E})$. This is obtained starting from the set of edges $\mathcal{E}$, removing a number of edges and keeping the unoriented edges $T \subseteq \mathcal{E}$. The unoriented edges that have been removed are the ones belonging to $\mathcal{E} \backslash T$. Consider now the metric graph $(V, \mathbf{E})$ and perform one single cut on each metric edge that is in correspondence with an unoriented edge in $\mathcal{E} \backslash T$. The metric graph that we obtain after all the cuts is then a metric tree.

Let $\mathcal{C}$ the collection of cutting points that transform the metric graph $(V, \mathbf{E})$ into a metric tree. When the dimension of the cut space is $k=|\mathcal{E} \backslash T|$ then a generic element of $\mathcal{C}$ is given by $y=\left(y_{1}, \ldots y_{k}\right)$ where each $y_{i}$ belongs to a different element of $\mathbf{E}$ associated to an edge in $\mathcal{E} \backslash T$. $\overline{W e}$ call $\mathbb{T}$ the collection of the spanning trees of $(V, \mathcal{E})$ and $\mathcal{C}(T)$ the cutting points compatible with the spanning tree $T \in \mathbb{T}$ as discussed above. We have therefore $\mathcal{C}=\cup_{T \in \mathbb{T}} \mathcal{C}(T)$. Given $\underline{y}=\left(y_{1}, \ldots, y_{k}\right) \in \mathcal{C}(T)$ we call $\tau_{x}[\underline{y}]$ the metric arborescence obtained cutting the metric graph on the points $\left(y_{1}, \ldots, y_{k}\right)$ and orienting the edges toward $x$.

Let us define a positive measure $m=\left(m_{\mathbf{e}}(x) d x\right)_{\mathbf{e} \in \mathbf{E}}$ on $\mathcal{G}$ that gives zero weight to vertices and that is absolutely continuous on the edges, defined by

$$
m_{\mathbf{e}}(x):=\sum_{T \in \mathbb{T}} \int_{\mathcal{C}(T)} d y_{1} \ldots d y_{k} R\left(\tau_{x}[\underline{y}]\right), \quad x \in \mathbf{e} .
$$

We have the following

Theorem 6.1. Under the condition

$$
W_{v}^{+}(\mathbf{e})=K_{v} \alpha_{v, \mathbf{e}}, \quad v \in V, \mathbf{e} \in A(v),
$$

where $K_{v}>0$ is a family of arbitrary constants, the positive measure $m$ defined by (6.3) satisfies conditions (1), (2) and (3A) in Lemma 5.3.

Proof: The basic fact to prove this theorem is the computation of

$$
\partial_{x}\left(\int_{\mathcal{C}(T)} d y_{1} \ldots d y_{k} e^{\int_{\tau_{x}[\underline{y}]} s}\right)
$$

We do it by distinguishing several cases. The first case is when $x \in \mathbf{e}$ and $\mathbf{e} \cap \mathcal{C}(T)=\emptyset$. In this case we can differentiate directly and using the computations in Lemma 3.3 we get that (6.5) is equal to

$$
\frac{2 b_{\mathbf{e}}(x)}{\sigma_{\mathbf{e}}^{2}(x)} \int_{\mathcal{C}(T)} d y_{1} \ldots d y_{k} e^{\int_{\tau_{x}[\underline{y}]} s} .
$$



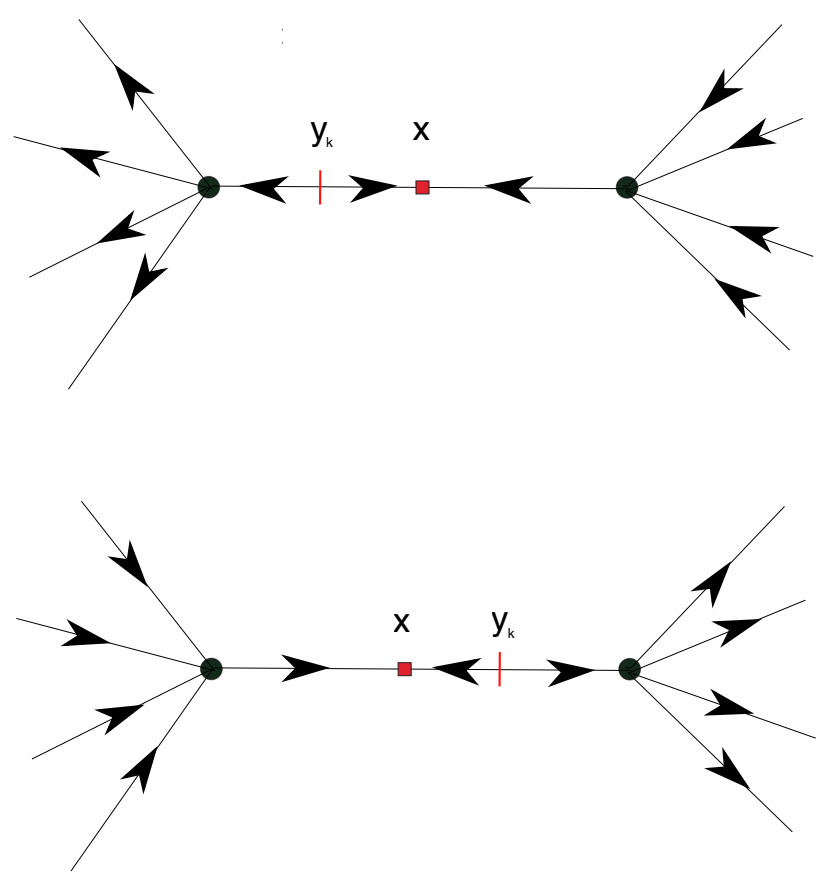

Figure 6.6. An edge in $\mathcal{C}(T)$ with the root $x$ denoted by a black cross and the cut point $y_{k}$ denoted by a red cross. In the upper part we illustrate the case $y_{k}<x$ while in the bottom part we illustrate the case $y_{k}>x$. The orientations outside of the edge containing $x$ are constant and depend just on the sign of the above inequalities. This corresponds to the fact that $\tau_{x}^{\prime}[y]$ assumes just the two configurations $\tau_{x \pm}^{\prime}$ letting vary $x$ on the edge.

In the case instead that $x \in \mathbf{e}$ and $\mathbf{e} \cap \mathcal{C}(T) \neq \emptyset$ we have to do a different computation. Let us assume (for simplicity of notation and without loss of generality) that $y_{k}$ is the cutting point that belongs to e. We can write the term to be differentiated in (6.5) as

$$
\begin{aligned}
\int_{\mathcal{C}^{\prime}(T)} d y_{1} \ldots d y_{k-1} e^{\int_{\tau_{x}^{\prime}[\underline{y}]} s}\left(\int_{0}^{x} d y_{k} e^{\int_{y_{k}}^{0} s_{\mathbf{e}}(z) d z} e^{\int_{y_{k}}^{x} s_{\mathbf{e}}(z) d z} e^{\int_{\ell_{\mathbf{e}}}^{x} s_{\mathbf{e}}(z) d z}\right. \\
\left.+\int_{x}^{\ell_{\mathbf{e}}} d y_{k} e^{\int_{0}^{x} s_{\mathbf{e}}(z) d z} e^{\int_{y_{k}}^{x} s_{\mathbf{e}}(z) d z} e^{\int_{y_{k}}^{\ell_{\mathbf{e}}} s_{\mathbf{e}}(z) d z}\right)
\end{aligned}
$$

In the above formula we called $\mathcal{C}^{\prime}(T)$ the cutting points that do not belong to e and we called $\tau_{x}^{\prime}[y]$ the metric graph obtained from $\tau_{x}[y]$ removing the oriented edges contained in e. First of all we observe that $\int_{\tau_{x}^{\prime}[\underline{y}]} s$ as a function of $x$ is piecewise constant and assumes only two values depending whether $x<y_{k}$ of $x>y_{k}$; we denote such values and the corresponding pieces of arborescences as

$$
\int_{\tau_{x}^{\prime}[\underline{y}]} s= \begin{cases}\int_{\tau_{x-[}^{\prime}[\underline{y}]} s & y_{k}<x, \\ \int_{\tau_{x+[}^{\prime}[\underline{y}]} s & y_{k}>x .\end{cases}
$$

See Figure 6.6 for a pictorial illustration of $\tau_{x}^{\prime}[\underline{y}]$. Let us call for simplicity of notation the two terms inside parenthesis in (6.7) as

$$
\int_{0}^{x} d y_{k} \psi_{-}\left(y_{k}, x\right)+\int_{x}^{\ell} d y_{k} \psi_{+}\left(y_{k}, x\right) .
$$


By a simple direct computation we have that $\partial_{x} \psi_{ \pm}\left(y_{k}, x\right)=2 s(x) \psi_{ \pm}\left(y_{k}, x\right)$, when $x \neq y_{k}$. We are therefore in the multidimensional counterpart of the situation discussed in Lemma 3.3. In particular we have to perform an $\epsilon$ type argument like the one just above formula (3.27) to deduce a result that is the multidimensional counterpart of (3.27). As in formula (3.27), we have in the derivation with respect to $x$, when $x \in \mathbf{e}$ with $\mathbf{e} \cap \mathcal{C}(T) \neq \emptyset$, the appearence of an extra term:

$$
\partial_{x} \int_{\mathcal{C}(T)} d y_{1} \ldots d y_{k} e^{\int_{\tau_{x}[\underline{y}]} s}= \begin{cases}2 s_{\mathbf{e}}(x) \int_{\mathcal{C}(T)} d y e^{\int_{\tau_{x}[\underline{y}]} s} & x \in \mathbf{e}, \mathbf{e} \cap \mathcal{C}(T)=\emptyset, \\ 2 s_{\mathbf{e}}(x) \int_{\mathcal{C}(T)} d y e^{\int_{\tau_{x}[\underline{y}} s}+\Delta & x \in \mathbf{e}, \mathbf{e} \cap \mathcal{C}(T) \neq \emptyset .\end{cases}
$$

The term $\Delta$ in the above equation can be computed as a limit like in formula (3.26), in particular we have

$$
\Delta=\Delta^{-}-\Delta^{+}
$$

where

$$
\begin{aligned}
\Delta^{-} & =\lim _{y_{k} \uparrow x} \int_{\mathcal{C}^{\prime}(T)} d y_{1} \ldots d y_{k-1} e^{\int_{\tau_{x}^{\prime}[\underline{y}]}^{s}} \psi_{-}\left(y_{k}, x\right) \\
& =\int_{\mathcal{C}^{\prime}(T)} d y_{1} \ldots d y_{k-1} e^{\int_{\tau_{x-}^{\prime}[\underline{y}]^{s}}^{s} e^{\int_{x}^{0} s} e^{\int_{\ell_{\mathbf{e}}}^{x} s}}
\end{aligned}
$$

and

$$
\begin{aligned}
\Delta^{+} & =\lim _{y_{k} \downarrow x} \int_{\mathcal{C}^{\prime}(T)} d y_{1} \ldots d y_{k-1} e^{\int_{\tau_{x}^{\prime}[\underline{y}]}^{s}} \psi_{+}\left(y_{k}, x\right) \\
& =\int_{\mathcal{C}^{\prime}(T)} d y_{1} \ldots d y_{k-1} e^{\int_{\tau_{x+}^{\prime}[\underline{y}]}^{s} e^{\int_{x}^{\ell_{\mathbf{e}}} s} e^{\int_{0}^{x} s} .}
\end{aligned}
$$

All the above computations can be done using the same argument used in the simpler framework in Lemma 3.3.

Moreover, we can offer some simple and natural interpretations of the above formulas. We have two contributions, one is positive while the other is negative. These contributions can be naturally interpreted as weights coming from arborescences that have a cut in the edge e that coincides exactly with the point $x$. The positive term $\Delta^{-}$corresponds to the weight of the arborescence when both parts of $\mathbf{e}$ are oriented in the opposite way with respect to the canonical one. The term with the minus sign $\Delta^{+}$corresponds instead to the situation when both parts of the edge e are oriented in agreement with the canonical orientation. We call $\tau_{x \pm}[y]$ the corresponding two different arborescences (see Figure 6.7 for an illustrative example) and notice that this notation is compatible with formula (6.8) as we now explain. Consider a cutting point $\underline{y}=\left(y_{1}, \ldots, x, \ldots, y_{k}\right)$ having a cut in correspondence of $x \in \mathbf{e}$. We write $\tau_{x-}^{\prime}[\underline{y}]$ to denote the part of the arborescence $\tau_{x-}[y]$ outside of the edge $\mathbf{e}$ when both parts of $\mathbf{e}$ are oriented in the opposite way with respect to the canonical one. We write instead $\tau_{x+}^{\prime}[y]$ to denote the part of the arborescence $\tau_{x+}[y]$ outside of the edge $\mathbf{e}$ when both parts of $\mathbf{e}$ are oriented in agreement with respect to the canonical one.

An alternative natural interpretation of the terms in (6.11) is the following. We call $\mathfrak{L}$ the set of uni-cyclic oriented metric spanning subgraphs of $(V, \mathbf{E})$ defined as follows. We call $\mathbb{L}$ the connected spanning subgraphs of $(V, \mathcal{E})$ that contains one single cycle and we call $\mathbb{L}_{\mathbf{e}} \subseteq \mathbb{L}$ the ones such that the element of $\mathcal{E}$ corresponding to $\mathbf{e}$ is one of the edges of the unique cycle. For any $L \in \mathbb{L}$ we call $\tilde{\mathcal{C}}(L)$ the sets of points $\tilde{y}=\left(y_{1}, \ldots, y_{k-1}\right) \in \mathbf{E}^{k-1}$ such that the $y_{i}$ belong to different metric edges and each of them corresponds to an element of $\mathcal{E}$ that has been erased from $(V, \mathcal{E})$ to get $L$. We call a uni-cyclic spanning metric subgraph $\mathcal{L} \in \mathfrak{L}$ the metric graph obtained from $(V, \mathbf{E})$ by the above cuts $\left(y_{1}, \ldots, y_{k-1}\right)$, such that all the edges are oriented toward the unique cycle and the edges of the cycle are oriented in such a way that it is possible to go around respecting the orientation. For each $\mathcal{L} \in \mathfrak{L}$ there exists a $\overline{\mathcal{L}} \in \mathfrak{L}$ such that $\mathcal{L}$ and $\overline{\mathcal{L}}$ are obtained by the same cuts, the edges outside of the cycle are oriented in the same way and all the edges inside the cycle are oriented in the opposite 

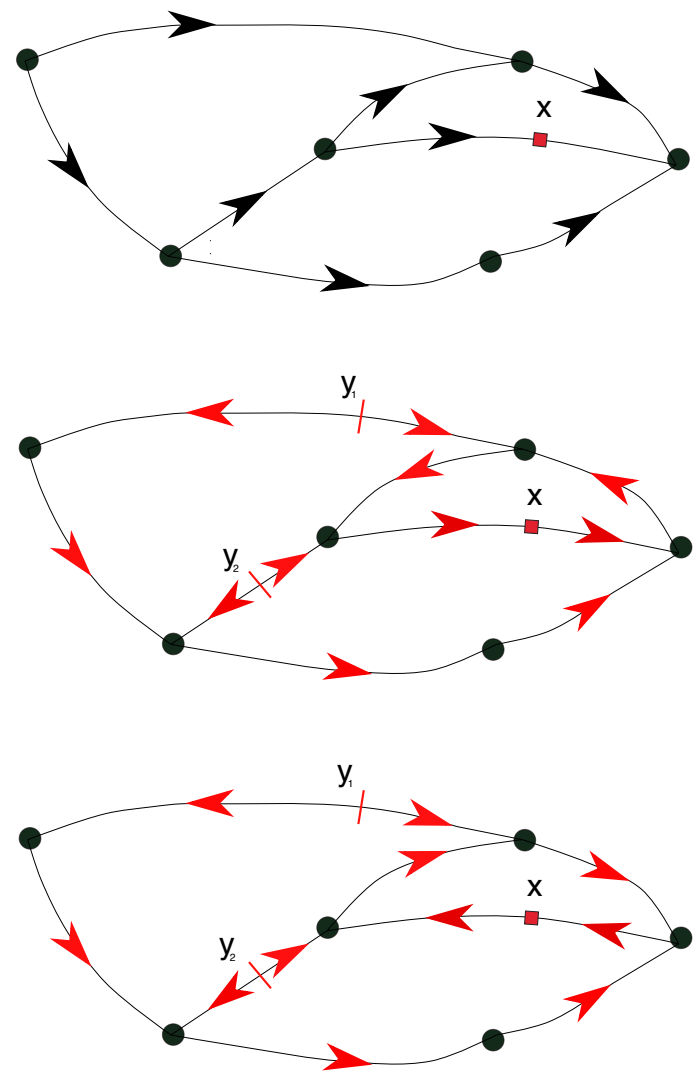

Figure 6.7. A metric graph with a marked point $\mathrm{x}$. The black arrows denote the canonical orientation of the edges (top picture). The oriented arborescence $\tau_{x+}(\underline{y})$ where the three cuts $y=\left(y_{1}, y_{2}, x\right)$ are represented respectively by two cutting red segments and a red $\mathrm{x}$ in correspondence of the marked point $\mathrm{x}$. The orientations of the branches of the arborescence are red colored (middle picture). The oriented arborescence $\tau_{x-}(y)$ where $y$ is as before. The orientations of the branches of the arborescence are red colored (bottom picture).

way. Given $\tilde{y} \in \tilde{\mathcal{C}}(L)$ we call $\mathcal{L}(\tilde{y}, \theta)$, with $\theta= \pm$ the two uni-cyclic spanning metric subgraphs with the two possible orientations of the cycle. Consider a metric arborescence $\tau_{x \pm}(\tilde{y}, x)$ obtained from $\mathcal{G}$ by a cutting set of the form $(\tilde{y}, x)$. A unicyclic metric graph $\mathcal{L}(\tilde{y}, \theta)$ is obtained from $\tau_{x \pm}(\tilde{y}, x)$ by removing the cut at $x$ and in this case $x$ belongs to the unique cycle (see Figure 6.8). We are now ready to do computations. Recalling formulas (6.1), (6.2) and (6.3) we have that

$$
\partial_{x}\left(\sigma_{\mathbf{e}}^{2}(x) m_{\mathbf{e}}(x)\right)=\sum_{T \in \mathbb{T}} \partial_{x}\left(\int_{\mathcal{C}(T)} d y_{1} \ldots d y_{k} e^{\int_{\tau_{x}[\underline{y}]} s} \prod_{v \in V} \mathcal{W}_{v}\left(\tau_{x}(\underline{y})\right)\right) .
$$

For simplicity of notation we did not consider the term $\prod_{v \in V} \mathcal{W}_{v}\left(\tau_{x}(\underline{y})\right)$ in the introductory computations. The reason is that the behavior of this term is the same of the term $e^{\int_{\tau_{x}^{\prime}[y]} s}$. This means that when $x \in \mathbf{e}$ with $\mathbf{e} \cap \mathcal{C}(T)=\emptyset$ then $\prod_{v \in V} \mathcal{W}_{v}\left(\tau_{x}(\underline{y})\right)$, as a function of $x$, is constant while when $x \in \mathbf{e}$ with $\mathbf{e} \cap \mathcal{C}(T) \neq \emptyset$ then $\prod_{v \in V} \mathcal{W}_{v}\left(\tau_{x}(\underline{y})\right)$, as a function of $x$, is piecewise constant and, in particular, it assumes only two values, namely one when $y_{k}<x$ and one when $y_{k}>x$. 

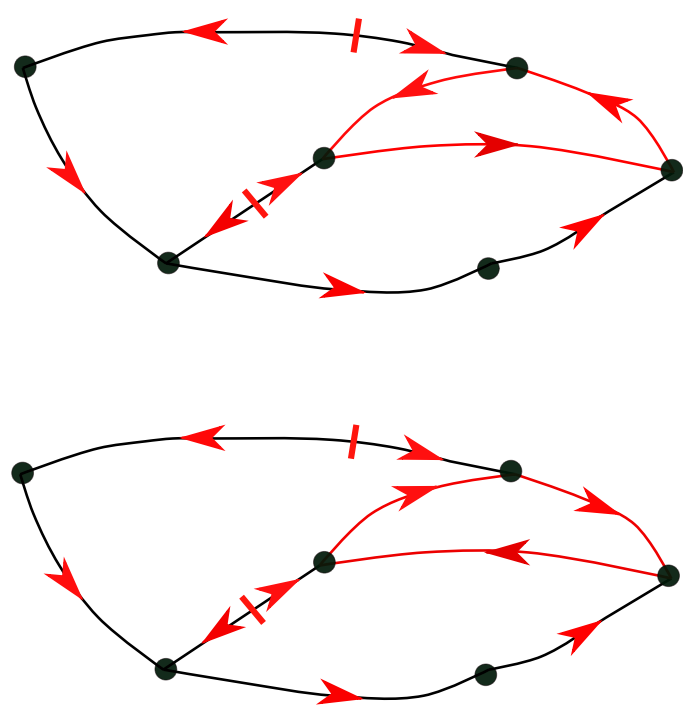

Figure 6.8. Two uni-cyclic spanning metric oriented subgraphs $\mathcal{L}$ and $\overline{\mathcal{L}}$ of the metric graph in the top picture of Figure 6.7. The orientations are red colored, the unique cycle is also red colored and oppositely oriented in the two pictures. Note that the two pictures are obtained by the middle and bottom pictures of Figure 6.7 simply removing the $\mathrm{x}$ cut.

We can therefore use formula (6.10), where we recall that we have to insert a proper factor containing the weights on the nodes. In particular, considering just the terms different from $\Delta$ in (6.10), we have in (6.12) a contribution given by

$$
2 b_{\mathbf{e}}(x) m_{\mathbf{e}}(x) .
$$

The remaining contributions are one positive, coming from the terms of the type $\Delta^{-}$and one negative, coming from the terms of the type $\Delta^{+}$. Given $x \in \mathbf{e}$, the positive one is given by

$$
\sum_{L \in \mathbb{L} \mathbf{e}} \int_{\tilde{\mathcal{C}}(L)} d y_{1}, \ldots d y_{k-1} e^{\int_{\mathcal{L}\left(\tilde{y}, \theta^{*}\right)} s} \prod_{v \in V} \mathcal{W}_{v}\left(\mathcal{L}\left(\tilde{y}, \theta^{*}\right)\right)
$$

In the above formula $\theta^{*}$ denotes the orientation of the unique cycle opposite to the natural one of $\mathbf{e}$ and the $\mathcal{W}$ terms give the weights to the nodes depending on the local orientation of the edges and they are defined like in (6.2). Given $x \in \mathbf{e}$, the one appearing with a negative sign in front is given by

$$
\sum_{L \in \mathbb{L} \mathbf{e}} \int_{\tilde{\mathcal{C}}(L)} d y_{1}, \ldots d y_{k-1} e^{\int_{\mathcal{L}\left(\tilde{y},-\theta^{*}\right)} s} \prod_{v \in V} \mathcal{W}_{v}\left(\mathcal{L}\left(\tilde{y},-\theta^{*}\right)\right)
$$

where $-\theta^{*}$ corresponds to the opposite orientation of the cycle with respect to $\theta^{*}$ and it agrees therefore with the natural one of $\mathbf{e}$. The above formulas are obtained by considering all the contributions of the terms of the type $\Delta^{ \pm}$and considering the interpretation in terms of unicyclic graphs of the different contributions. In particular formulas (6.14) and (6.15) are obtained according to our previous discussion by the following identification. Consider an $x \in \mathbf{e}$ and denote by $\sum_{T \in \mathbb{T}}^{*}$ the sum over all spanning trees that do not contain the edge corresponding to the metric edge e. Equivalently the set of cut points must satisfy $\mathcal{C}(T) \cap \mathbf{e} \neq \emptyset$. Like in formula (6.7) we call $\mathcal{C}^{\prime}(T)$ the cut 
points that do not belong to e. Formulas (6.14) and (6.15) are obtained by the formal identification $\sum_{T \in \mathbb{T}}^{*} \int_{\mathcal{C}^{\prime}(T)}=\sum_{L \in \mathbb{L} \mathbf{e}} \int_{\tilde{\mathcal{C}}(L)}$, i.e. summing over the two classes of graphs and integrating in those classes of cut points is equivalent. The arguments to be integrated in formulas (6.14) and (6.15) are obtained recalling that unicyclic metric graphs $\mathcal{L}(\tilde{y}, \theta)$ are obtained from $\tau_{x \pm}(\tilde{y}, x)$ by removing the cut at $x$ and in this case $x$ belongs to the unique cycle (see Figure 6.8).

We can now prove the properties of the measure $m$.

(1): Recall that the current probability along each edge is given by

$$
J_{\mathbf{e}}[m]:=-\frac{1}{2} \partial_{x}\left(\sigma_{\mathbf{e}}^{2}(x) m_{\mathbf{e}}(x)\right)+b_{\mathbf{e}}(x) m_{\mathbf{e}}(x) .
$$

If we insert the three terms obtained by the computation of $\partial_{x}\left(\sigma_{\mathbf{e}}^{2}(x) m_{\mathbf{e}}(x)\right)$ we obtain the following. The term (6.13) matches exactly twice the last term in (6.16) and due to the factor $1 / 2$ they cancel. This means that the probability current is exactly equal to minus $1 / 2$ the difference of the two terms in (6.14) and (6.15). A key observation is now the following. Formulas (6.14) and (6.15) hold for any $x \in \mathbf{e}$, however as it is apparent from the explicit form there is a dependence on $\mathbf{e}$ but not a dependence on the specific point $x \in \mathbf{e}$. This means that these two terms do not depend on the specific point $x \in \mathbf{e}$ and we have that the current (6.16) is therefore constant along each edge.

(2): The divergence free condition is obtained by the following argument. Let us write shortly formulas (6.14) and (6.15) as $\sum_{L \in \mathbb{L}_{\mathbf{e}}} \Delta_{\mathbf{e}}^{ \pm}(L)$. We obtain that, for any $x \in \mathbf{e}$, the probability current assumes the same value:

$$
J_{\mathbf{e}}[m]=\frac{1}{2} \sum_{L \in \mathbb{L}_{\mathbf{e}}}\left(\Delta_{\mathbf{e}}^{+}(L)-\Delta_{\mathbf{e}}^{-}(L)\right) .
$$

Given $L \in \mathbb{L}$ we define $J[L]$ as

$$
J_{\mathbf{e}}[L]:= \begin{cases}\Delta_{\mathbf{e}}^{+}(L)-\Delta_{\mathbf{e}}^{-}(L) & L \in \mathbb{L}_{\mathbf{e}} \\ 0 & L \notin \mathbb{L}_{\mathbf{e}}\end{cases}
$$

With these definitions we have that $J[m]=\frac{1}{2} \sum_{L \in \mathbb{L}} J[L]$ and since the divergence is a linear operator it is enough to prove that each $J[L]$ is divergence free. Note that $J[L]$ is different from zero only on the edges that belong to the unique cycle of $L$. Consider a fixed $L \in \mathbb{L}_{\mathbf{e}}$ and a metric edge $\mathbf{e}^{\prime}$ in correspondence with one edge belonging to the unique cycle of $L$. This means that we have also $L \in \mathbb{L}_{\mathbf{e}^{\prime}}$. Consider $\mathbf{e}$ and $\mathbf{e}^{\prime}$ having the vertex $v$ in common (see Figure 6.9). A key observation that is obtained by the explicit formulas is the following. If the natural orientations of the two metric edges $\mathbf{e}$ and $\mathbf{e}^{\prime}$ are compatible with the same global orientation of the unique cycle of $L$ then $\Delta_{\mathbf{e}}^{+}(L)-\Delta_{\mathbf{e}}^{-}(L)=\Delta_{\mathbf{e}^{\prime}}^{+}(L)-\Delta_{\mathbf{e}^{\prime}}^{-}(L)$. If the natural orientations of the two metric edges $\mathbf{e}$ and $\mathbf{e}^{\prime}$ are instead compatible with opposite global orientations of the unique cycle of $L$ then we have $\Delta_{\mathbf{e}}^{+}(L)-\Delta_{\mathbf{e}}^{-}(L)=\Delta_{\mathbf{e}^{\prime}}^{-}(L)-\Delta_{\mathbf{e}^{\prime}}^{+}(L)$. It is now immediate to verify the divergence free condition at vertex $v$. The same argument can be done for any vertex in the cycle. Since outside of the cycle $J[L]$ is zero, we have that in the remaining vertices the divergence free condition is automatically satisfied.

$(3 A)$ : In the computation of $m_{\mathbf{e}}(v)$ we can ignore, when considering the limit $y \rightarrow v$ in (5.3), the contribution from integrations coming from cuts in a neighborhood of the node $v$. More precisely consider $\mathbf{e}, \mathbf{e}^{\prime} \in A(v)$ with $v \in V$ and $z \in \mathbf{e}, z^{\prime} \in \mathbf{e}^{\prime}$ such that $z, z^{\prime}$ are at distance (along the edges of the metric graph) $\epsilon$ from $v$. We can write

$$
m_{\mathbf{e}}(z)=\sum_{T \in \mathbb{T}} \int_{\overline{\mathcal{C}}^{\epsilon}(T)} d y_{1} \ldots d y_{k} R\left(\tau_{z}[y]\right)+\sum_{T \in \mathbb{T}} \int_{\mathcal{C}^{\epsilon}(T)} d y_{1} \ldots d y_{k} R\left(\tau_{z}[y]\right)
$$

where $\mathcal{C}^{\epsilon}(T)$ is the collection of cuts such that there is at least one cut at distance less or equal to $\epsilon$ from $v$ while $\overline{\mathcal{C}}^{\epsilon}(T)$ is the complementary set, i.e. the collection of cuts that are all at distance 


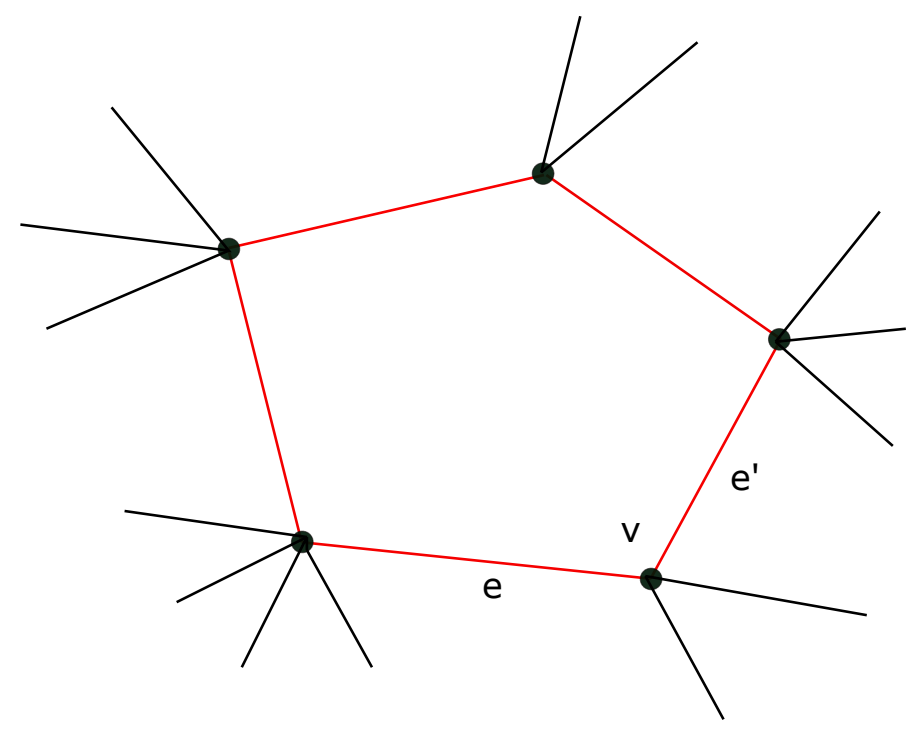

Figure 6.9. We draw in red the unique cycle in $L$ and give an example of a pair of metric edges $\mathbf{e}$ and $\mathbf{e}^{\prime}$ belonging to this cycle and sharing a vertex $v$.

greater that $\epsilon$ from $v$. A formula similar to (6.17) can be written also for the point $z^{\prime}$. In the limit $\epsilon \rightarrow 0$ the second term in (6.17) is negligible.

The basic observation is the following. Recall that by definition, in an arborescence, when the root $x$ does not coincide with a vertex we have that exactly one edge is oriented exiting from the vertex while all the others are oriented entering into the vertex. This is because otherwise we could find two different paths from the vertex to the root. See for example Figure 4.5 where it is possible to check the validity of this fact on all the vertices. Consider a tree $T$ and some cutting points $\underline{y} \in \overline{\mathcal{C}}^{\epsilon}(T)$. In this case all the edges $\mathbf{e}^{\prime \prime} \in A(v)$ such that $\mathbf{e}^{\prime \prime} \neq \mathbf{e}$ will be oriented locally around $\bar{v}$ in $\tau_{z}[y]$ entering into $v$ while instead e will be oriented exiting from $v$. In $\tau_{z^{\prime}}[y]$ we have instead that $\mathbf{e}^{\prime}$ is oriented exiting from $v$ while instead all the other edges in $A(v)$ will be oriented entering into $v$. In particular in $\tau_{z^{\prime}}[y]$ and $\tau_{z}[y]$ all the branches of the two arborescenses will have the same orientation apart the two segments $(v, z) \subseteq \mathbf{e}$ and $\left(v, z^{\prime}\right) \subseteq \mathbf{e}^{\prime}$ that are of size $\epsilon$.

We have therefore that for any $T$ and for any $\underline{y} \in \overline{\mathcal{C}}^{\epsilon}(T)$

$$
\frac{R\left(\tau_{z}(\underline{y})\right)}{R\left(\tau_{z^{\prime}}(\underline{y})\right)}=e^{2 \int_{x}^{z} s} e^{2 \int_{z^{\prime}}^{x} s} \frac{W_{x}^{+}(\mathbf{e}) \sigma_{\mathbf{e}^{\prime}}^{2}\left(z^{\prime}\right)}{W_{x}^{+}\left(\mathbf{e}^{\prime}\right) \sigma_{\mathbf{e}}^{2}(z)} .
$$

Taking the limit $\epsilon \rightarrow 0$ in (6.18) and using (6.4) we finish the proof.

Using the above result we can finally conclude. Let us call

$$
\tilde{\lambda}_{v}:=\lim _{y \in \mathbf{e}, y \rightarrow v} \frac{\sigma_{\mathbf{e}}^{2}(y) m_{\mathbf{e}}(y)}{\alpha_{v, \mathbf{e}}} \quad v \in V, \mathbf{e} \in A(v) .
$$

We observe by Theorem 6.1 that $\tilde{\lambda}_{v}$ does not depend on the edge $\mathbf{e} \in A(v)$. We have indeed that by Theorem 6.1 the measure $m$ satisfies condition $(3 A)$ of Lemma 5.3 and the constants $\tilde{\lambda}_{v}$ here are the constants $\lambda_{v}$ appearing in condition $(3 A)$. Let us also introduce the probability measure $\mu$ as in (5.4) defined by

$$
\begin{cases}\mu_{v}:=\frac{\tilde{\lambda}_{v} \alpha_{v}}{Z} & v \in V \\ \mu_{\mathbf{e}}(x):=\frac{m_{\mathbf{e}}(x)}{Z} & x \in \mathbf{e}\end{cases}
$$


where $Z$ is the normalization constant determined by (5.5).

Theorem 6.2. The probability measure (6.20) is the invariant measure of the process with generator satisfying (5.1), provided that (6.4) holds.

Proof: Conditions (1) and (2) in Lemma 5.3 depend just on the form of the measure $\mu$ on the edges and they are preserved by a multiplication of the measure by a constant factor. Since by Theorem 6.1 the measure $m$ satisfies these conditions and since the density of $\mu$ on each edge is obtained multiplying the density of $m$ by the factor $Z^{-1}$ we deduce that $\mu$ satisfies conditions (1) and (2).

Condition (3) on Lemma 5.3 is satisfied by the fact that the limit on the right hand side of (6.19) does not depend on $\mathbf{e} \in A(v)$ and by definition (6.20).

Since conditions (1), (2) and (3) characterize the unique invariant measure we deduce that $\mu$ is the invariant measure.

This construction of the invariant measure gives as special cases formula (3.23) for a one dimensional ring, and formula (3.7) in the case of a single interval since in that case the cut space is empty and there are no integrations to be done.

6.1. The reversible case: In the reversible case all the structure simplifies and the invariant measure can be simply computed in terms of an auxiliary finite state Markov chain on the set $V$. The characterization of reversibility was discussed also in Jehring (2009), Fitzsimmons and Kuter (2015) in terms of a similar discrete time Markov chain that is the process associated to the sequence of visited nodes. We start defining our auxiliary Markov chain. Given $v, w \in V$ we define the rate of jump from $v$ to $w$ across the edge $\mathbf{e} \in A(v) \cap A(w)$ as

$$
q(v, w):= \begin{cases}\alpha_{v, \mathbf{e}} e^{\int_{0}^{\ell_{\mathbf{e}}} s_{\mathbf{e}}} & \text { if } \mathbf{e} \in A^{+}(v) \cap A^{-}(w), \\ \alpha_{v, \mathbf{e}} e^{-\int_{0}^{\ell_{\mathbf{e}}} s_{\mathbf{e}}} & \text { if } \mathbf{e} \in A^{-}(v) \cap A^{+}(w) .\end{cases}
$$

For simplicity we restrict to the case $|A(v) \cap A(w)| \leq 1$. The general case can be discussed very similarly.

We have the following characterization of reversible processes and their invariant measures.

Theorem 6.3. Consider a diffusion process on a metric graph $\mathcal{G}$ having generator $\mathcal{A}$ as in Definition 5.1. Then the process is reversible if and only if the Markov chain on $V$ with rates (6.21) is reversible. In this case the invariant measure of the process is given by

$$
\mu= \begin{cases}\mu_{\mathbf{e}}(x)=\frac{2 c \pi_{v} q(v, w)}{\sigma_{\mathbf{e}}^{2}(x)} e^{\int_{0}^{x} s_{\mathbf{e}}(y) d y-\int_{x}^{\ell_{\mathbf{e}}} s_{\mathbf{e}}(y) d y}, & x \in \mathbf{e} \in A(v) \cap A(w), \\ \mu_{v}=c \pi_{v} \alpha_{v}, & v \in V,\end{cases}
$$

where $\left(\pi_{v}\right)_{v \in V}$ is the unique invariant measure of the Markov chain with rates (6.21) and $c$ is a suitable normalization constant.

Proof: Suppose that the Markov chain with rates (6.21) is reversible with invariant measure $\pi$. Then by the detailed balance relationship

$$
\pi_{v} q(v, w)=\pi_{w} q(w, v)
$$

the measure $\mu$ is well defined. We have to show that the measure (6.22) satisfies the conditions $\left(1^{\prime}\right)$ and (3) in Lemma 5.3. Condition $\left(1^{\prime}\right)$ is satisfied since on each edge the measure is given by the upper formula in (6.22) that coincides, by (3.28) (3.29), with the simple case (3.7) that corresponds to zero probability current.

If we compute the quantities appearing in condition (3), that is formula (5.7), we obtain

$$
\begin{cases}\frac{1}{2} \sigma_{\mathbf{e}}^{2}(v) \mu_{\mathbf{e}}(v)=c \pi_{v} \alpha_{v, \mathbf{e}} & v \in V, \mathbf{e} \in A(v) \\ \mu_{v}=c \pi_{v} \alpha_{v} & v \in V\end{cases}
$$


so that condition (3) is satisfied with $\lambda_{v}=c \pi_{v}$.

Conversely suppose that the diffusion process on a metric graph having generator $\mathcal{A}$ as in Definition 5.1 is reversible. Then, since the probability current across each edge must be zero, the density of the invariant measure $\mu$ on each edge has to be of the form

$$
\mu_{\mathbf{e}}(x)=\frac{C_{\mathbf{e}}}{\sigma_{\mathbf{e}}^{2}(x)} e^{\int_{0}^{x} s_{\mathbf{e}}(y) d y-\int_{x}^{\ell} s_{\mathbf{e}}(y) d y},
$$

for some suitable constants $C_{\mathbf{e}}$ (formula (6.24) is the general solution of the equation $J_{\mathbf{e}}[\mu]=0$, as follows by the identity (3.29)). Condition (3) for the stationarity implies that there exists some positive numbers $\left(\lambda_{v}\right)_{v \in V}$ for which we have

$$
\begin{cases}C_{\mathbf{e}}=\lambda_{v} \alpha_{v, \mathbf{e}} e^{\int_{0}^{\ell_{\mathbf{e}}} s_{\mathbf{e}}(y) d y}, & \text { if } \mathbf{e} \in A^{+}(v) \\ C_{\mathbf{e}}=\lambda_{v} \alpha_{v, \mathbf{e}} e^{-\int_{0}^{\ell_{\mathbf{e}}} s_{\mathbf{e}}(y) d y}, & \text { if } \mathbf{e} \in A^{-}(v) .\end{cases}
$$

Take $\mathbf{e} \in A(v) \cap A(w)$ and compute $C_{\mathbf{e}}$ using (6.25) using the two different formulas at $v$ and $w$. We get

$$
C_{\mathbf{e}}=\lambda_{v} q(v, w)=\lambda_{w} q(w, v) .
$$

Normalising to one the sum of $\lambda$ 's we have that the second identity above coincides with the detailed balance for the Markov chain with rates $q$.

We remark that the simple case discussed in Remark 3.4 of the interval with reflecting boundary conditions always corresponds to a reversible situation since it is associated to a two states Markov chain.

\section{Acknowledgments}

We thank an anonymous referee for a very careful reading of the paper which led to a notable improvement of the text.

\section{References}

Anantharam, V. and Tsoucas, P. A proof of the Markov chain tree theorem. Statist. Probab. Lett., 8 (2), 189-192 (1989). MR1017890.

Andreucci, D., Cirillo, E. N. M., Colangeli, M., and Gabrielli, D. Fick and Fokker-Planck diffusion law in inhomogeneous media. J. Stat. Phys., 174 (2), 469-493 (2019). MR3910901.

Bakry, D., Gentil, I., and Ledoux, M. Analysis and geometry of Markov diffusion operators, volume 348 of Grundlehren der Mathematischen Wissenschaften [Fundamental Principles of Mathematical Sciences]. Springer, Cham (2014). ISBN 978-3-319-00226-2; 978-3-319-00227-9. MR3155209.

Barlow, M., Pitman, J., and Yor, M. On Walsh's Brownian motions. In Séminaire de Probabilités, XXIII, volume 1372 of Lecture Notes in Math., pp. 275-293. Springer, Berlin (1989). MR1022917.

Berkolaiko, G., Carlson, R., Fulling, S. A., and Kuchment, P., editors. Quantum graphs and their applications, volume 415 of Contemporary Mathematics. American Mathematical Society, Providence, RI (2006). ISBN 0-8218-3765-6. MR2279143.

Bogachev, V. I., Krylov, N. V., Röckner, M., and Shaposhnikov, S. V. Fokker-Planck-Kolmogorov equations, volume 207 of Mathematical Surveys and Monographs. American Mathematical Society, Providence, RI (2015). ISBN 978-1-4704-2558-6. MR3443169.

Burago, D., Burago, Y., and Ivanov, S. A course in metric geometry, volume 33 of Graduate Studies in Mathematics. American Mathematical Society, Providence, RI (2001). ISBN 0-8218-2129-6. MR1835418.

Camilli, F. and Marchi, C. A comparison among various notions of viscosity solution for HamiltonJacobi equations on networks. J. Math. Anal. Appl., 407 (1), 112-118 (2013). MR3063108. 
Colangeli, M., Maes, C., and Wynants, B. A meaningful expansion around detailed balance. J. Phys. A, 44 (9), 095001, 13 (2011). MR2771859.

Faggionato, A. and Gabrielli, D. A representation formula for large deviations rate functionals of invariant measures on the one dimensional torus. Ann. Inst. Henri Poincaré Probab. Stat., 48 (1), 212-234 (2012). MR2919204.

Fitzsimmons, P. J. and Kuter, K. E. Harmonic functions of Brownian motions on metric graphs. J. Math. Phys., 56 (1), 013504, 28 (2015). MR3390843.

Freidlin, M. and Sheu, S.-J. Diffusion processes on graphs: stochastic differential equations, large deviation principle. Probab. Theory Related Fields, 116 (2), 181-220 (2000). MR1743769.

Freidlin, M. I. and Wentzell, A. D. Diffusion processes on graphs and the averaging principle. Ann. Probab., 21 (4), 2215-2245 (1993). MR1245308.

Freidlin, M. I. and Wentzell, A. D. Random perturbations of Hamiltonian systems. Mem. Amer. Math. Soc., 109 (523), viii+82 (1994). MR1201269.

Freidlin, M. I. and Wentzell, A. D. Random perturbations of dynamical systems, volume 260 of Grundlehren der Mathematischen Wissenschaften [Fundamental Principles of Mathematical Sciences]. Springer, Heidelberg, third edition (2012). ISBN 978-3-642-25846-6. MR2953753.

Garavello, M. and Piccoli, B. Traffic flow on networks. Conservation laws models, volume 1 of AIMS Series on Applied Mathematics. American Institute of Mathematical Sciences (AIMS), Springfield, MO (2006). ISBN 978-1-60133-000-0; 1-60133-000-6. MR2328174.

Gardiner, C. Stochastic methods. A handbook for the natural and social sciences. Springer Series in Synergetics. Springer-Verlag, Berlin, fourth edition (2009). ISBN 978-3-540-70712-7. MR2676235.

Hajri, H. and Raimond, O. Stochastic flows on metric graphs. Electron. J. Probab., 19, no. 12, 20 (2014). MR3164765.

Jehring, K. E. Harmonic functions on Walsh's Brownian motion. ProQuest LLC, Ann Arbor, MI (2009). ISBN 978-1109-15160-2. Thesis (Ph.D.)-University of California, San Diego. MR2713145.

Kostrykin, V., Potthoff, J., and Schrader, R. Brownian motions on metric graphs. J. Math. Phys., 53 (9), 095206, 36 (2012). MR2905788.

Mugnolo, D. Semigroup methods for evolution equations on networks. Understanding Complex Systems. Springer, Cham (2014). ISBN 978-3-319-04620-4; 978-3-319-04621-1. MR3243602.

Norris, J. R. Markov chains, volume 2 of Cambridge Series in Statistical and Probabilistic Mathematics. Cambridge University Press, Cambridge (1998). ISBN 0-521-48181-3. MR1600720.

Pavliotis, G. A. Stochastic processes and applications. Diffusion processes, the Fokker-Planck and Langevin equations, volume 60 of Texts in Applied Mathematics. Springer, New York (2014). ISBN 978-1-4939-1322-0; 978-1-4939-1323-7. MR3288096.

Pitman, J. and Tang, W. Tree formulas, mean first passage times and Kemeny's constant of a Markov chain. Bernoulli, 24 (3), 1942-1972 (2018). MR3757519.

Walsh, J. B. A diffusion with a discontinuous local time. Astérisque, 52 (53), 37-45 (1978). 